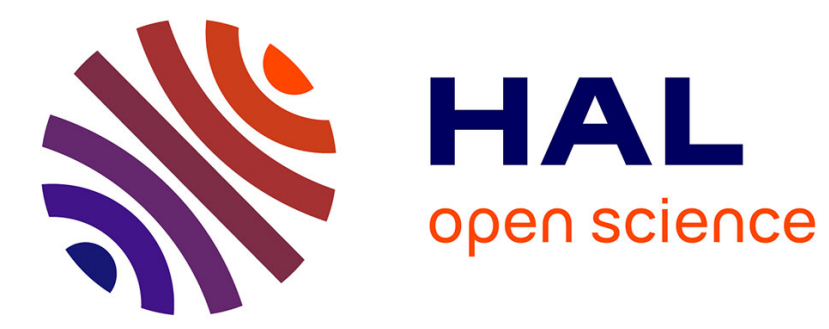

\title{
A Multi-Model View of Process Modelling
}

Colette Rolland, Naveen Prakash, Adolphe Benjamen

\section{To cite this version:}

Colette Rolland, Naveen Prakash, Adolphe Benjamen. A Multi-Model View of Process Modelling. Requirements Engineering, 1999, pp.169 - 187. hal-00707568

\section{HAL Id: hal-00707568 https://hal.science/hal-00707568}

Submitted on 16 Jun 2012

HAL is a multi-disciplinary open access archive for the deposit and dissemination of scientific research documents, whether they are published or not. The documents may come from teaching and research institutions in France or abroad, or from public or private research centers.
L'archive ouverte pluridisciplinaire HAL, est destinée au dépôt et à la diffusion de documents scientifiques de niveau recherche, publiés ou non, émanant des établissements d'enseignement et de recherche français ou étrangers, des laboratoires publics ou privés. 


\title{
A multi-model view of process modelling
}

\author{
Colette Rolland*, Naveen Prakash ${ }^{+}$, Adolphe Benjamen* \\ Université de Paris1 Sorbonne \\ Centre de Recheche en Informatique (CRI) \\ 17 , rue de la Sorbonne \\ 75231 Paris cedex 05 \\ France \\ *\{rolland,benjamen $\} @$ univ-paris1.fr \\ +np@dit.ernet.in
}

\begin{abstract}
Situatedness of development processes is a key issue in both the software engineering and the method engineering communities, as there is a strong felt need for process prescriptions to be adapted to the situation at hand. The assumption of the process modelling approach presented in this paper is that process prescriptions shall be selected according to the actual situation at hand i.e. dynamically in the course of the process. The paper focuses on a multi-model view of process modelling which supports this dynamicity. The approach builds on the notion of a labelled graph of intentions and strategies called a map as well as its associated guidelines. The map is a navigational structure which supports the dynamic selection of the intention to be achieved next and the appropriate strategy to achieve it whereas guidelines help in the operationalization of the selected intention. The paper presents the map and guidelines and exemplifies the approach with the CREWS-L'Ecritoire* method for requirements engineering.
\end{abstract}

\section{Introduction}

Process engineering is considered today as a key issue by both the software engineering and information systems engineering communities. Recent interest in process engineering is part of the shift of focus from the product to the process view of systems development. The belief of the software engineering community is that as a result of improved development processes [Dow93], [Arm93] and [Jar94]. there shall be both, improved productivity of the software systems industry and improved systems quality, The focus has been to increase the level of formality of process models in order to make possible their enactment in Process Centred Software Environments [Fin94]. As a

\footnotetext{
* This work is partly funded by the Basic Research Action CREWS (ESPRIT N ${ }^{\circ} 21.903$ ). CREWS stands for Cooperative Requirements Engineering With Scenarios
} 
consequence a large number of process models have been developed that Dowson [Dow93] classifies as activity-oriented models, product-oriented models and decisionoriented models.

The software process modelling community realised quite early that even though process models were prescriptive, in actual practice departures from the prescription occurred [Hid94], [Rus95], [Wij90], [Aae92] and [You92]. Therefore, a concerted effort was put in to allow process models to respond to these departures. One approach was to assume prescriptive models and then, modify them to accommodate real processes. This modification could be achieved in two ways. First the extent of deviations from the prescription that could be allowed was modelled as constraints [Cug95, Cug96, Cug98]. Any actual deviation that satisfied the constraint was therefore manageable and the process enactment mechanism could handle it. This way of handling deviations took the prescriptive approach to its logical conclusion : it prescribed the deviations allowed in a prescription. The second way of handling deviations is to allow changes to be made in the prescription as and when they are needed [Dow94, SiS96, Jac92, Fin94, Ban93, Be194]. Thus, a dynamic change of the basic prescription is allowed.

In recent years, the information systems community has concentrated on the need for adapting and extending existing methods to meet the changing needs of practice. Method engineering [We192], [Har94] represents the effort to improve the usefulness of systems development methods by creating an adaptation framework whereby methods are created to match specific organisational situations. This improvement has been attempted at two levels. At a global level, it deals with determining the project contingency factors [Slooten], [Euromethod] that help in selecting the right method to be used whereas at a more fine-grained level it deals with on-the-fly construction of the process prescription fitting the situation at hand.

The latter was carried out in the contextual model [Gha97, Ro195, Poh96, Bub94]. Here the attempt was to relax the prescription given by a process model. Thus, the process model did not always specify what must be done but contained some specification of what can be done. The process model therefore, contained a number of alternative ways of doing a task and a selection of the particular alternative was done dynamically, depending upon the situation in which the product was found. However, the contextual model could consist of both alternatives as well as prescriptions. Whenever such alternatives were available, the net effect was that the process model could be dynamically built, even as the process was being performed. The major difference between the software engineering approaches and the contextual approach is that 
whereas handling departures from prescriptions is an exception handling activity in the former, selection from alternatives in the latter is the normal activity envisaged in the process model itself and supported by a dynamic selection mechanism. Thus, support for real processes is provided in a more natural way.

In this paper, we propose to relax the prescription of a process model even further. Our proposal is based on the experience with the contextual model that we gained working with four groups of postgraduate students. The experiment consists of using the six methods described with the contextual model in [Pli94] to develop application case studies within the process centred environment MENTOR [SiS96]. Our experience was that a key discriminant factor in real processes is the product situation. This situation has a strong bearing in selecting the task best suited to handle it and also the strategy to be adopted in carrying out this task. For example, consider a process for doing requirements engineering using goal-scenario coupling. Assume that a goal $\mathrm{G}$ has been elicited. Now, it is possible to either explore alternative goals of $\mathrm{G}$ or to write a scenario for it. Thus, the process model must reflect this choice and the requirements engineer would dynamically choose between one of these alternatives. It can be seen that $\mathrm{G}$ provides a basis for a discriminant choice in what task is to be done next. Now, consider that a fully developed scenario has been written out and goals are to be determined by scenario analysis. That is, the next task to be done is known. However, it is possible to discover goals that are exceptions or obstacles to $G$ or sub-goals of $G$ using the alternative or the composition discovery strategies. Again, these strategies for eliciting goals need to be reflected in the process model so that the right one can be dynamically chosen depending on the nature of the scenario. Thus, the product situation also provides a basis for a discriminant choice in what strategy is to be adopted in performing a task. Evidently, a process model that captures all alternatives of tasks and strategies is needed to support processes. Such a model needs to be backed up by a dynamic selection mechanism of tasks and strategies. In the paper we propose to represent task and strategies alternatives as a labelled directed graph called a map and provide support in alternative selection through guidelines.

It can be seen that the salient features of our approach are

i) explicit recognition of the role of strategies in process modelling,

ii) a non-prescriptive model of strategies and tasks containing alternatives only from which real processes can be built,

iii) dynamic process construction is the rule rather than an exception. 
As indicated above, the non-prescriptive model is a labelled directed graph called a map. The map uses two fundamental notions, a process intention or intention for brevity, and strategy. An intention captures in it the notion of a task that the application engineer intends to perform whereas the strategy is the manner in which the intention can be achieved. The nodes of the map are intentions whereas the edges are labelled with strategies. The directed nature of the map identifies which intention can be done after a given one. The only way in which a process can be built is dynamically, through the use of guidelines for selection among alternatives. Only after the task and the strategy have been decided is there a need for a guideline to achieve the task.

There are three guidelines associated with the map :

- intention selection guidelines for determining all succeeding intentions of a given one, - strategy selection guidelines for determining the strategies from which one is selected, - intention achievement guidelines for defining the way in which an intention can be achieved. Thereafter, the enactment mechanism is invoked to actually carry out the tasks.

We view a map as containing a panel of process prescriptions from which, by dynamic selection, the particular one that is best suited to the product situations as they emerge is selected. In this sense, the map is a multi-model with dynamic process modelling capability.

The layout of the paper is as follows. In the next section the notion of the map as a labelled directed graph is presented and the multi-model capability of the map is highlighted. In section III, the different kinds of guidelines and their structure are considered. The manner in which guidelines relate to the map is articulated. Section IV contains the representation of the CREWS-L'Ecritoire method as a map of guidelines. This serves as an example to illustrate how the map and guidelines can be used to represent real methods. Section V deals with the meta-process i.e. the process to develop and enact application processes. The use of the meta-process to develop the requirements specification of a recycling machine is presented in section VI. Section VII is the concluding section.

\section{The Map}

A map is a process model which is associated with a product model as shown in Figure 1 to form a method. Figure 1 describes our method view using an E/R like notation. A box represents an Entity Type (ET), the labelled link represents a Relationship Type 
(RT) and the embedded box refers to an objectified RT. Multiplicities are denoted with couples of minimum and maximum cardinality values.

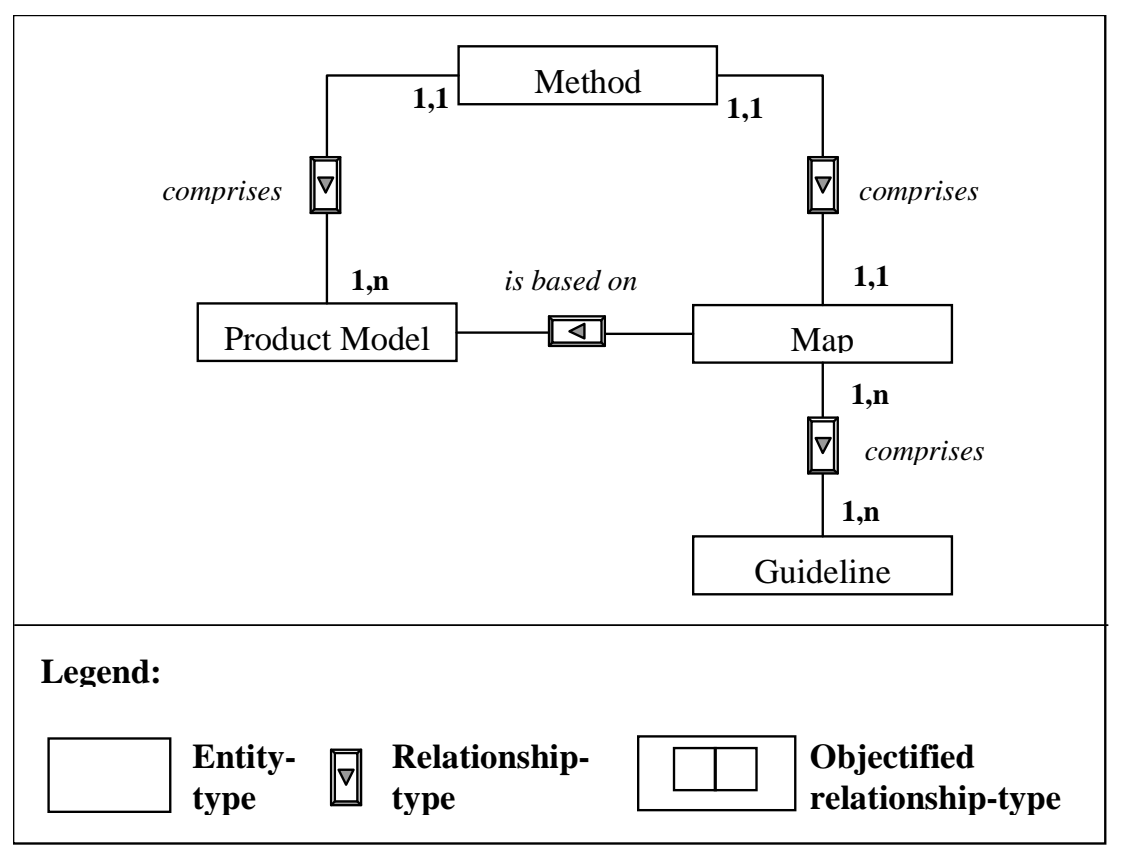

Figure 1: Map and Product model

A map is a process model in which a non-deterministic ordering of intentions and strategies has been included. It is a labelled directed graph with intentions as nodes and strategies as edges between intentions. The directed nature of the graph shows which intentions can follow which one. Figure 2 describes the map meta-model using the same E/R like notation as above. As shown in the figure, a map consists of a number of sections each of which is a triplet $\left\langle I^{1}{ }_{i}, I_{j}, \mathrm{~S}^{2}{ }_{\mathrm{ij}}\right\rangle$. There are two distinct intentions called Start and Stop respectively that represent the intentions to start navigating in the map and to stop doing so. Thus, it can be seen that there are a number of paths in the graph from Start to Stop.

\footnotetext{
${ }^{1}$ Intention are in italics $\left(I_{i}, I_{j}\right)$

${ }^{2}$ Strategies are in " arial " $\left(\mathrm{S}_{\mathrm{ij}}\right)$
} 


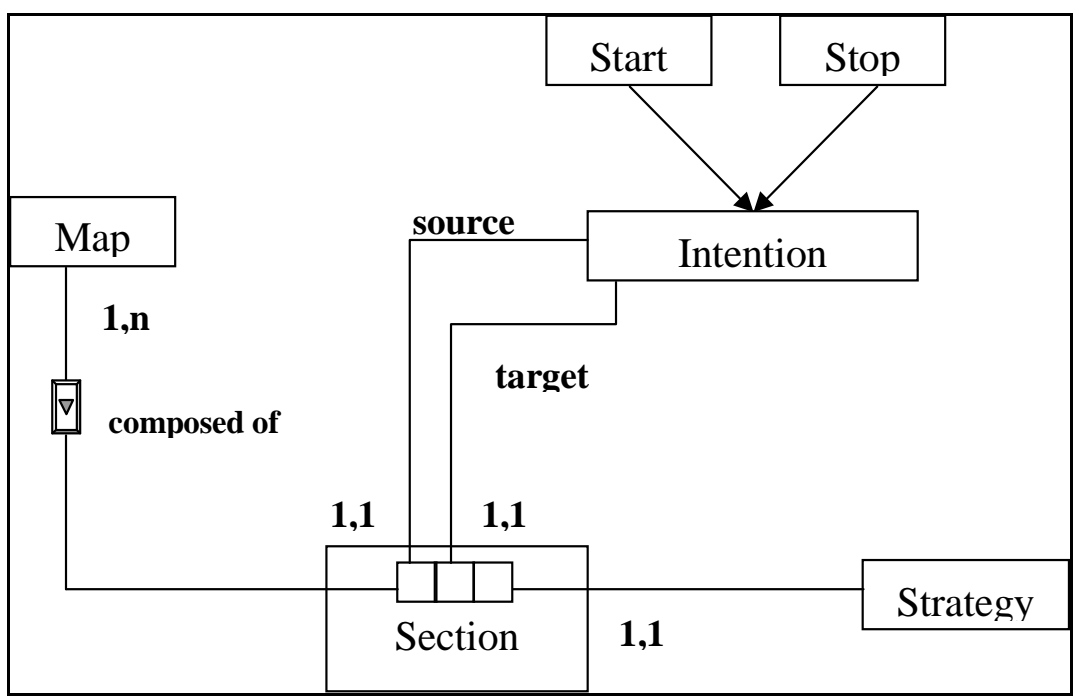

Figure 2: The map meta-model

We assume development processes to be intention-oriented. At any moment, the application engineer has an intention, a goal in mind that he/she wants to fulfil. To take this characteristic into account the map identifies the set of intentions that have to be achieved in order to solve the problem at hand.

\section{Let I be this set.}

An intention is a goal, an objective that the application engineer has in mind at a given point of time. An intention statement expressed in natural language usually starts with a verb and may comprise several parameters, where each parameter plays a different role with respect to the verb. The key parameter is the target of the verb; for example in the examples below, Scenario and Goal are the targets of the verbs Conceptualize and Elicit respectively.
(a) Conceptualize verb a Scenario object
(b) Elicit verb $a$ Goal result $_{\text {to }}$

As shown in the examples above, there are two types of targets, Objects and Results. Both refer to product parts i.e. elements of the product model, which are either objects or subjects of the process intention. An Object is supposed to exist before the goal is achieved. For example in the goal statement (a) the target Scenario is an object because it exists even before Conceptualize is achieved. In contrast, a Result results of the achievement of the intention. For example in the goal statement (b), a Goal is the result of the achievement of the intention Elicit. We shall introduce other parameters of the verb in an intention statement as needed in the paper. For more details see [Pra97, Rol98b]. 
A strategy is an approach, a manner to achieve an intention. The strategy, as part of the triplet $\left\langle I_{i}, I_{j}, \mathrm{~S}_{\mathrm{ij}}\right\rangle$ characterizes the flow from $I_{i}$, to $I_{j}$ and the way $I_{j}$ can be achieved.

\section{Let $\mathrm{S}$ be the set of strategies identified in the map.}

It can be seen that the map can represent in it all the meaningful interconnections between process intentions and strategies. Formally, the map is a subset of the Cartesian product:

\section{$\mathbf{M a p} \subseteq \mathbf{I} \times \mathbf{I} \times \mathbf{S}$}

The specific manner in which an intention can be achieved is captured in a section of the map whereas the various sections having the same intention $I_{i}$ as a source and $I_{j}$ as target show the different strategies that can be adopted for achieving $I_{j}$ when coming from $I_{i}$. Similarly, there can be different sections having $I_{i}$ as source and $I_{j 1}, I_{j 2}, \ldots . I_{j n}$ as targets. These show the different intentions that can be achieved after the achievement of $I_{i}$.

Let there be two map sections, MS1 and MS2. MS1 and MS2 are connected in the map provided the target intention of MS1 is the source intention of MS2. For example, the sections $\left\langle I_{i}, I_{j}, \mathrm{~S}_{\mathrm{ij}}\right\rangle$ and $\left\langle I_{k}, I_{i}, \mathrm{~S}_{\mathrm{ki}}\right\rangle$ are interconnected in the map because the target intention $I_{i}$ of the latter is also the source intention of the former. Thus, $I_{j}$ is reachable from $I_{k}$ through the intermediate intention $I_{i}$.

As an example consider Figure 3 which contains six sections MS0 to MS5 having connections at $I_{i}, I_{j}$ and $I_{k}$.

As shown in the figure, there might be several flows from $I_{i}$ to $I_{j}$, each corresponding to a specific strategy (for examples MS1 and MS2 in Figure 3). In this sense the map offers multi-thread flows. There might also be several strategies from different intentions to reach an intention $I_{i}$ (for examples MS3 and MS4 in Figure 3). In this sense the map offers multi-flow paths to achieve an intention. Finally, the map can include reflexive flows (see MS3 in Figure 3). 


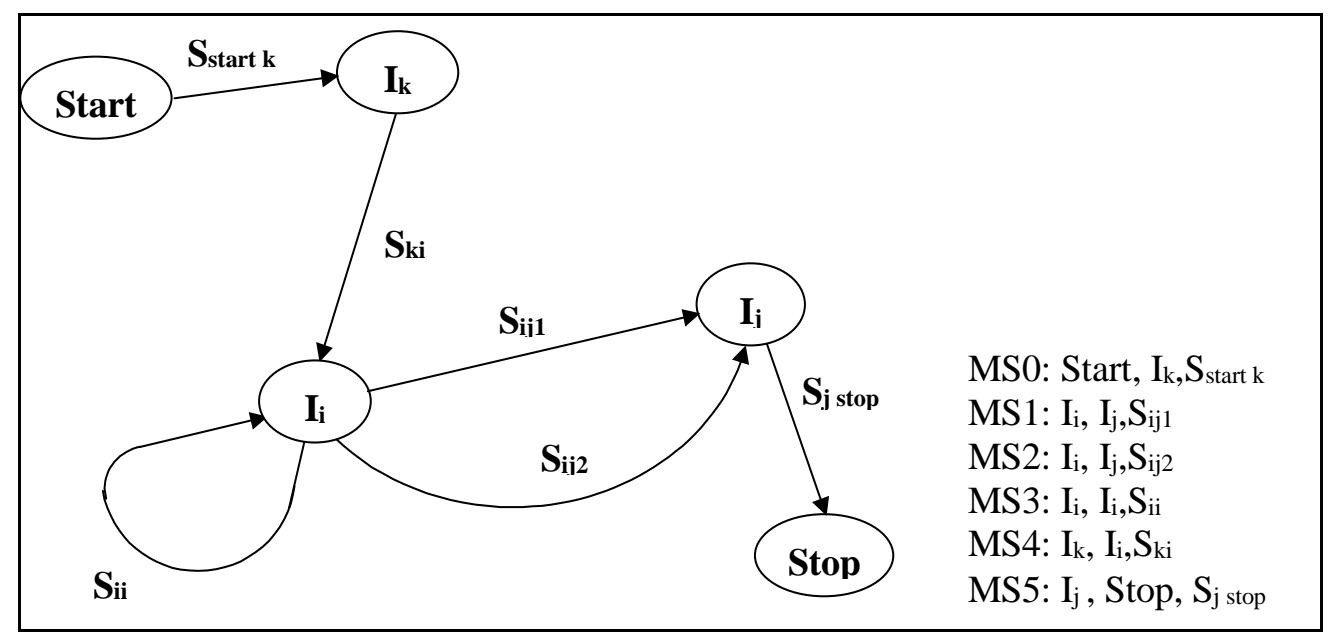

Figure 3: Examples of map sections

A map is a navigational structure in the sense that it allows the application engineer to determine a path from Start intention to Stop intention. The map contains a finite number of paths, each of them prescribing a way to develop the product i.e. each of them is a process model. Therefore the map is a multi-model. It embodies several process models, providing a multi-model view for modelling a class of processes. None of the finite set of models included in the map is recommended "a priori". Instead the approach suggests a dynamic construction of the actual path by navigating in the map. In this sense the approach is sensitive to the specific situations as they arise in the process. The next intention and strategy to achieve it are selected dynamically by the application engineer among the several possible ones offered by the map. Furthermore the approach is meant to allow the dynamic adjunction of a path in the map i.e adding a new strategy or a new section in the actual course of the process.

In such a case guidelines that make available all choices open to handle a given situation are of great convenience. The map is associated to such guidelines. These are presented in the next section.

\section{Guidelines}

A guideline is defined [LPR95] as 'a set of indications on how to proceed to achieve an objective or perform an activity'. For us, a guideline embodies method knowledge to guide the application engineer in achieving an intention in a given situation. In this section we first consider the different kinds of guidelines and their relationships to the map. Thereafter the structure of the guidelines as comprising a signature and a body is considered and the relationship between the guideline signature and the kind of guideline is brought out. 


\section{III.1 Kinds of Guidelines}

As shown in Figure 4, we associate the map with guidelines, namely one 'Intention Achievement Guideline' per section $\left\langle I_{i}, I_{j}, \mathrm{~S}_{\mathrm{ij}}\right\rangle$, one 'Intention Selection Guideline' per node $I_{i}$, except for Stop and one 'Strategy Selection Guideline' per node pair $\left\langle I_{i}, I_{j}\right\rangle$.We will refer to them as IAG, ISG and SSG respectively.

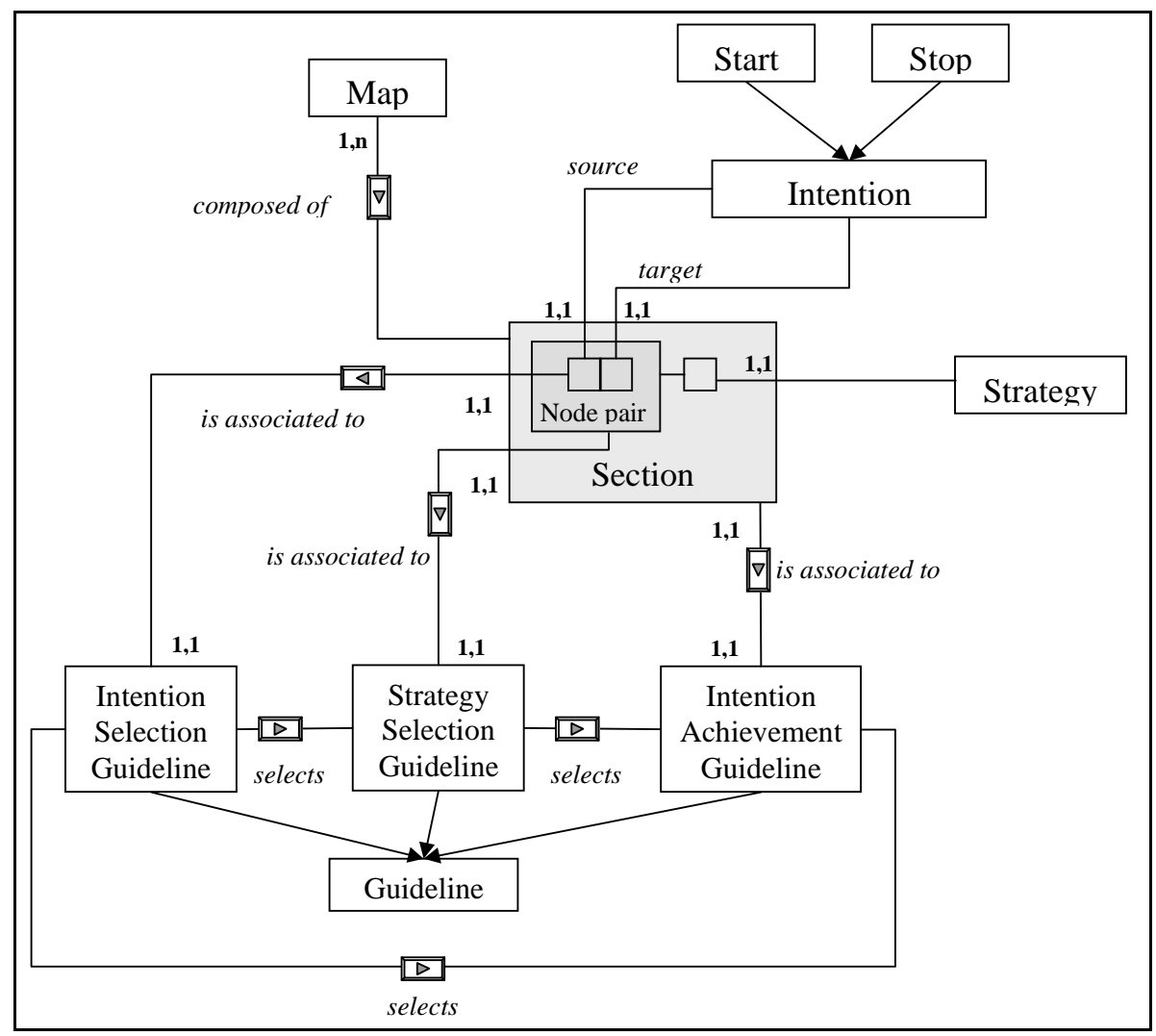

Figure 4: The map guideline relationships

An intention driven process is an iterative process that repeatedly resolves two issues, namely, (1) how to fulfil the intention he/she reached and (2) how to select the right section to progress. IAGs support the former whereas ISGs and SSGs help in the latter. More precisely:

(1) There exists an Intention Achievement Guideline (IAG) for every triplet $\left\langle I_{i}, I_{j}, \mathrm{~S}_{\mathrm{ij}}\right\rangle$. It aims at supporting the application engineer in the achievement of intention $I_{j}$ according to the strategy $S_{i j}$.

\section{For a section $\left\langle I_{i}, I_{j}, S_{i j}\right\rangle$, there is an IAG.}

An IAG provides an operational means to fulfil the intention. This means that an IAG implies the transformation of the product under development. Whereas the map 
identifies strategies to reach intentions, IAGs are concerned with the tactics to implement these strategies. There might be several tactics offered by an IAG. This means that an IAG may contain alternative operational ways to fulfil the intention. Besides it might be necessary to proceed in a number of steps to reach the ultimate effect of an IAG, that is to perform some action on the product under development. Consequently an IAG may include the decomposition of the initial intention into subintentions which themselves may be decomposed till intentions executable through actions on the product are reached. Therefore, an IAG may be seen as a goal tree which helps in performing the operationalization of an intention I through sub-intentions connected by alternative and decomposition relationships into actions on the product.

(2) Given two Intentions $I_{i}, I_{j}$ and a set of possible strategies $\mathrm{S}_{\mathrm{ij} 1}, \mathrm{~S}_{\mathrm{ij} 2}, . . \mathrm{S}_{\mathrm{ijn}}$ applicable to $I_{j}$, the role of the Strategy Selection Guideline ( $S S G$ ) is to guide the selection of an $\mathrm{S}_{\mathrm{ijk}}$ thereby leading to the selection of the corresponding IAG.

\section{For a node pair $\left\langle I_{i}, I_{j}\right\rangle$, there is an SSG.}

An SSG, first determines all the strategies that can be used to achieve $I_{j}$ from $I_{i}$. It does this by the operation SOP, Strategy Operator, defined as follows:

\section{SOP : $\mathbf{I} \times \mathbf{I} \rightarrow\{\mathbf{S} \mid<\mathbf{I}, \mathbf{I}, \mathbf{S}>$ is a section $\}$}

For example in the map of Figure 3

$$
\operatorname{SOP}\left(I_{i}, I_{j}\right)=\left\{\mathrm{S}_{\mathrm{ij} 1}, \mathrm{~S}_{\mathrm{ij} 2}\right\}
$$

The set of strategies is presented by SSG to the application engineer who picks the one most appropriate to the situation at hand. Thus, the section $\left\langle I_{i}, I_{j}, S_{\mathrm{ijk}}\right\rangle$ is selected. Since a unique Intention Achievement Guideline is associated with each section, the SSG determines this. The enactment mechanism then performs $I_{j}$ according to the selected strategy in the task organization specified by the Intention Achievement Guideline.

(3) Given an intention $I_{i}$, an Intention Selection Guideline (ISG), identifies the set of intentions $\left\{I_{j}\right\}$ that can be achieved in the next step and selects the corresponding set of either IAGs or SSGs. The former is valid when there is only one section between $I_{i}$ and $I_{j}$ whereas the latter occurs when there are several sections between $I_{i}$ and $I_{j}$.

For an intention $I_{i}$, there is an ISG. 
An ISG, first determines all the intentions that can be done after a given one. It does this through the operation IOP, Intention Operator, defined as follows:

\section{IOP : $\mathbf{I} \rightarrow\{\mathbf{I} \mid<\mathrm{I}, \mathrm{I}, \mathrm{S}>$ is a section $\}$}

That is, IOP determines the set of intentions which are the target intentions of sections having the same source intention.

For example, in the map of Figure 3:

$$
\operatorname{IOP}\left(I_{i}\right)=\left\{I_{j}, I_{i}\right\}
$$

The application engineer then picks up one intention out of these, the one which is most appropriate for the situation at hand. The ISG then determines whether there is only one section between the source and the selected target intention or whether there are several sections. In the former case, the IAG associated with the section is used by the enactment mechanism to achieve the target intention. In the case when several sections exist between the source and the selected target intention, the SSG is invoked to determine the strategy to be used in the situation which, as discussed earlier, leads to the determination of an IAG and subsequent enactment. In our example, IOP has determined two target intentions $I_{j}$ and $I_{i}$ as shown above. There is only one section between the source intention $I_{i}$ and the target $I_{i}$. This is $\left\langle I_{i}, I_{i}, \mathrm{~S}_{\mathrm{ii}}\right\rangle$. Thus, if the application engineer chooses $I_{i}$ as the target then, the IAG is determined. ISG can cause intention achievement with no further intervention from the application engineer. On the other hand, there are two sections having $I_{i}$ as source and $I_{j}$ as target. These are $\left\langle I_{i}, I_{j}, \mathrm{~S}_{\mathrm{ij} 1}\right\rangle$ and $\left\langle I_{i}, I_{j}, \mathrm{~S}_{\mathrm{ij} 2}\right\rangle$ respectively. If the application engineer chooses $I_{j}$ as the target intention then SSG must be used to decide which of these shall be used. The IAG is determined and $I_{j}$ achieved.

It can be seen from the foregoing that the objective of the ISGs is met by placing reliance upon SSGs and IAGs. Similarly SSGs rely on IAGs. Therefore, determination of the intention to handle a given situation, determination of the strategy to be adopted and the task organization are all integrated together.

Summarising then, Figure 5 below associates the ISGs, IAGs and SSGs with the map shown in Figure 3. There are six IAGs, one per section, four ISGs for each of the nodes except Stop, and four SSGs for each of the four node pairs $\left\langle I_{i}, I_{j}\right\rangle$. 


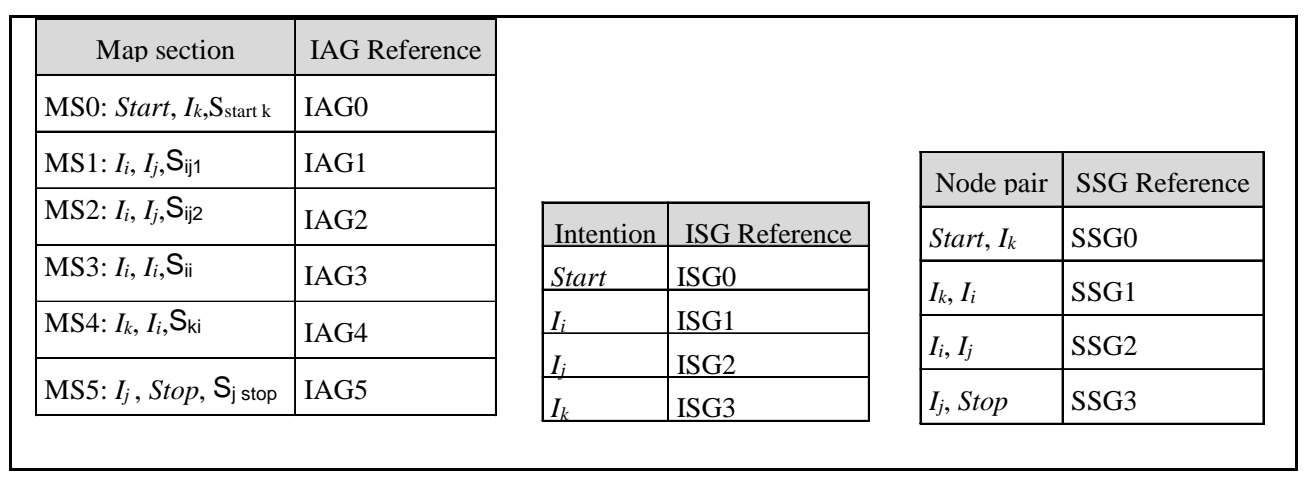

Figure 5 : Guidelines of the Map presented in Figure 3

\section{III.2 Structure of a Guideline}

Even though there are different kinds of guidelines, all of these depict the same underlying structure. Figure 6 shows the guideline meta-model expressed again in an E/R like notation. Our proposal for the description of a guideline relies on the NATURE contextual approach [Ro195, Gro97] and its corresponding enactment mechanism [SiS96, SiS97]. As shown in Figure 6, a guideline has a body which encapsulates method knowledge and a signature. We consider these in turn.

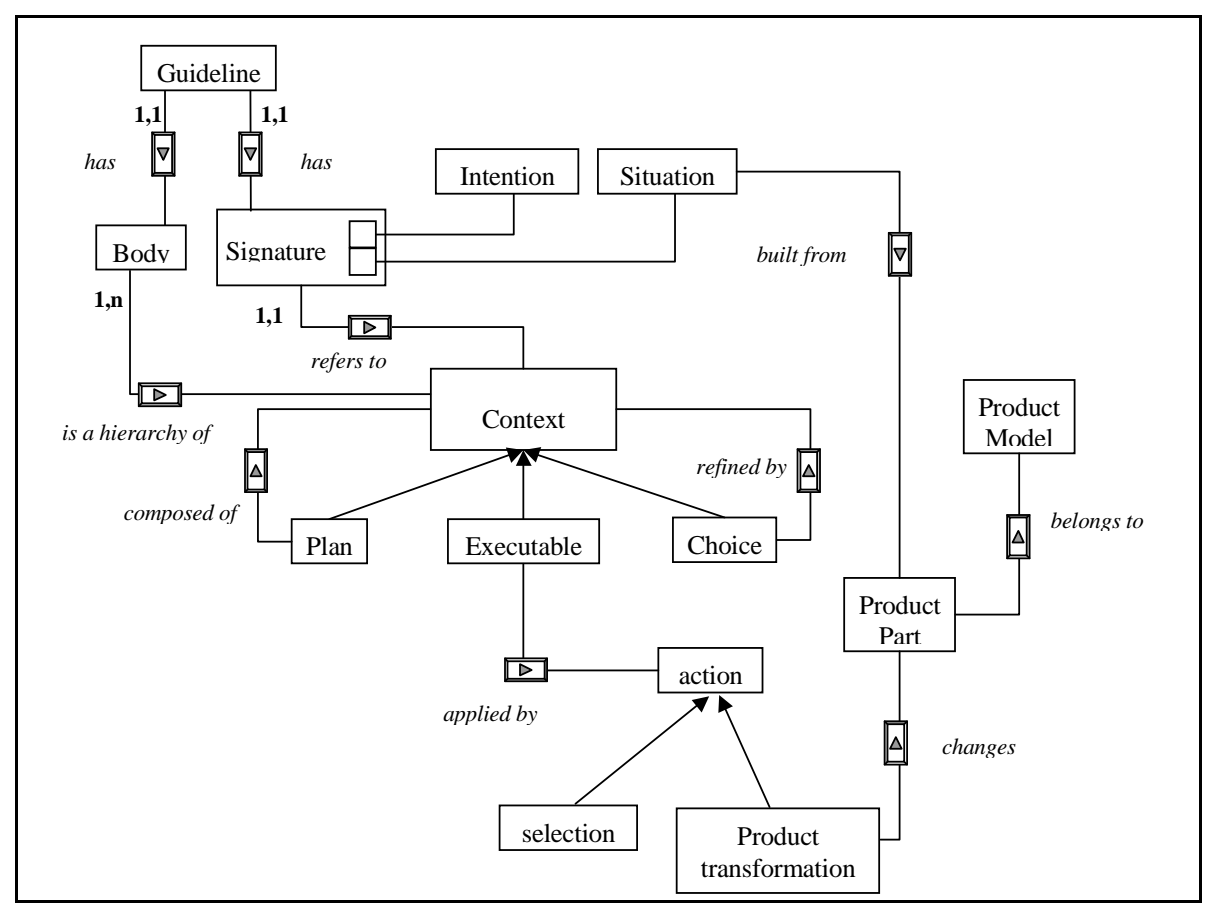

Figure 6: The guideline meta-model

\section{Guideline signature}

A signature is a pair $\langle$ (sit), $I\rangle$ where (sit) is the situation and $I$ is an intention. For example, <(Goal), Author Scenario $>$ is a signature. The situation refers to the product 
under development and the intention is the goal that the application engineer wants to achieve in this situation. In the previous example the situation is the product part 'Goal' and Author Scenario is the intention $I$ that the application engineer wants to achieve. The three kinds of guidelines namely ISGs, SSGs and IAGs have signatures of the generic form $\langle$ (sit), $I>$. However (sit) and $I$ can be specialized for each of the three kinds of guidelines. This is summed up in Figure 7 and explained below.

\begin{tabular}{|l|l|l|}
\hline Type of guideline & \multicolumn{1}{|c|}{ Map reference } & \multicolumn{1}{c|}{ Guideline signature } \\
\hline $\mathrm{IAG}_{\mathrm{i}}$ & $\left\langle I_{i}, I_{i}, \mathrm{~S}_{\mathrm{ij}}\right\rangle$ & $\left(\right.$ sit* $\left.^{*}\left(I_{i}\right), I_{j}\right)$ \\
\hline $\mathrm{ISG}_{\mathrm{i}}$ & $\left\langle I_{i}\right\rangle$ & $\left(\right.$ sit $\left(I_{i}\right)$, Progress from $\left.I_{i}\right)$ \\
\hline $\mathrm{SSG}_{\mathrm{i}}$ & $\left\langle I_{i}, I_{j}\right\rangle$ & $\left(\right.$ sit $\left(I_{i}\right)$, Progress to $\left.I_{j}\right)$ \\
\hline
\end{tabular}
*Sit $\left(I_{i}\right)$ refers to the product situation after $I_{i}$ has been achieved.
$\begin{aligned} & \text { Progress refers to a class of intentions in order to progress in the process. } \\
& \text { In contrast } I_{j}, I_{i} \text { are achievement intentions. }\end{aligned}$

\section{Figure 7: Correspondence between the kind of guideline and the guideline signature}

First, as mentioned earlier, the map identifies two issues to be solved by the application engineer (a) how to perform the intention he/she has reached and (b) how to select the right section to progress further. This leads to an identification of two major classes of intentions of signatures, the Achieve and the Progress. As IAGs support issue (a), the signature intention of a IAG refers to a process achievement intention and therefore belongs to the Achieve signature intention class. SSGs and ISGs which help in (b) have signature intentions which express process progression towards process achievement and therefore, belong to the Progress signature intention class. Therefore, we propose to use the map intention $I$ in IAG intention signatures and the generic term Progress as intention signature for SSGs and ISGs.

Second, we propose to differentiate an SSG intention signature from an ISG one using the statement Progress verb $\left(\text { from } I_{i}\right)_{\text {source }}$ for the former and Progress verb $\left(\text { to } I_{j}\right)_{\text {target }}$ for the latter.

Progress $_{\text {verb }}$ (from Author Scenario) source $_{\text {and }}$

Progress verb (to Author Scenario) $)_{\text {target }}$

are two examples of signature intentions belonging to the class Progress. As shown in these examples, Progress is the verb of the intention statement, (from Author Scenario) is the source parameter of the verb and (to Author Scenario) corresponds to the target parameter.

Third, we suggest to integrate the name of the strategy in the statement of the 
achievement intention of a IAG. Therefore, the IAG for a section $\left\langle I_{i}, I_{j}, S_{i j 1}\right\rangle$ has an intention signature of the form $I_{j}$ with $\mathrm{S}_{\mathrm{ij}}$.

Author $_{\text {verb }}$ Scenario result $_{\text {(with linguistic strategy) manner }}$

is an example of intention belonging to the class Achieve. As indicated in the intention statement Author is the verb, Scenario is its result and (with linguistic strategy) corresponds to the parameter manner.

Finally, the situation part of the guideline signature refers to the product part(s) resulting from the achievement of the start intention $\left(I_{i}\right)$ of the map section associated to the guideline. We will see in the next section that the situation may include constraints on the product. These constraints on (sit) play the role of a pre-condition for the intention $I$ to be achievable. It can be seen that the guideline establishes the connection between the process and the product models making precise the part of the product (and its associated constraints) influencing the process flow.

$($ Scenario $)$ and $($ Scenario: state $($ Scenario $)=$ written $)$

are two examples of situations. In the first case (Scenario) refers to the product part 'Scenario' whereas in the second case, the situation constrains the 'Scenario' to be in the state 'written'.

\section{Guideline body}

The body describes the way in which Achieve and Progress intentions are fulfilled. Following the contextual approach the body is organized around the notion of a context that can be of three different types: executable, plan, choice and two types of relationships among contexts: composition and refinement (Figure 6). The latter leads to an organization of a guideline as a hierarchy of contexts connected by AND (composed of) and OR (refined by) relationships. The former helps in distinguishing situations offering choices (choice contexts) from those which require decomposition of contexts (plan contexts). Executable contexts are of two types : in IAGs they are associated to actions which transform the product under development. The guideline is therefore a means to articulate the consequences of satisfying the intention of the guideline signature on the product under development. In SSGs and ISGs they perform actions to select IAGs. The enactment mechanism takes care of the presentation of available choices, the performance of plan contexts and of the impact of the execution of actions on the product under construction For further details on the contextual approach see [Ro193, Ro194a, Ro194b, Sut97, Ro195].

\section{A multi-model view of CREWS-L'Ecritoire}


This section instantiates the map meta-model presented in section 2 with the goalscenario method for Requirements Engineering developed in the CREWS project [Ben98, Rol97, Rol98b, Hau98]. The method combines a goal driven approach to requirement engineering with the use of scenarios. The total solution is in two parts. First, for a goal, scenarios are authored by the scenario author. Thereafter, the authored scenario is explored to yield goals which in turn, cause new scenarios to be authored and so on.

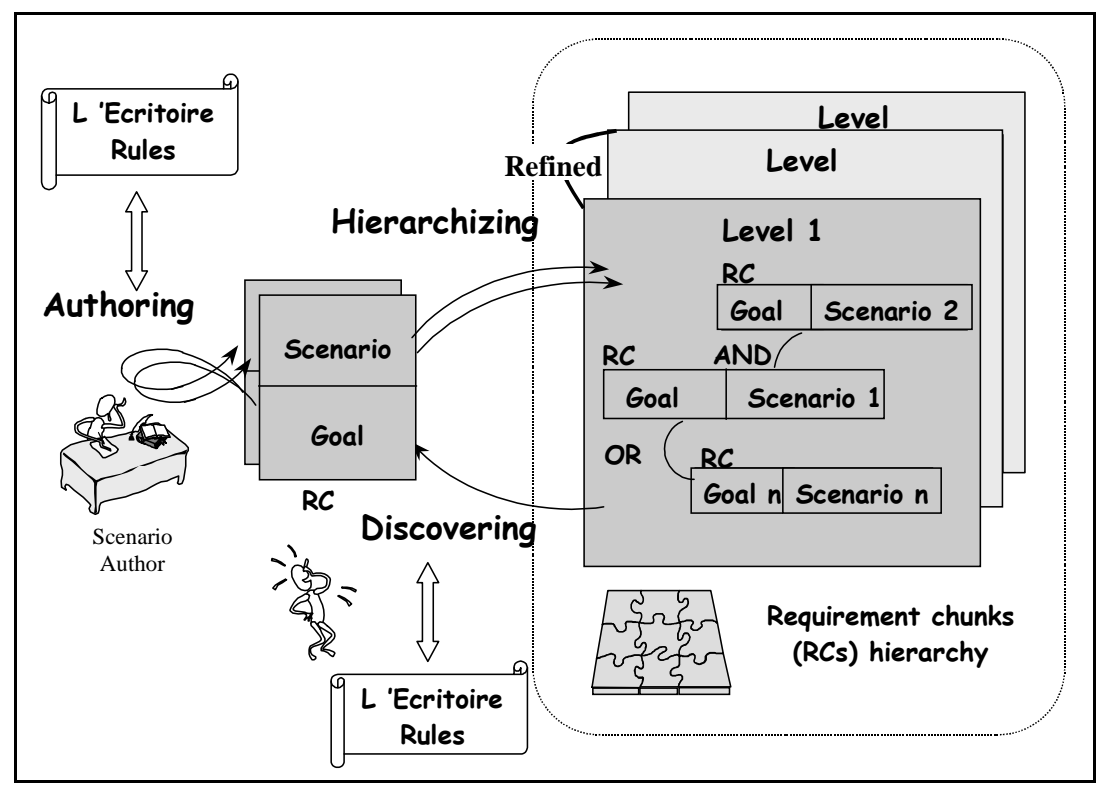

Figure 8: Overview of the CREWS RE process

As illustrated in Figure 8 the RE process consists of repeating a two-phase cycle composed of (1) scenario authoring and (2) goal discovery. The resulting product is a hierarchy of pairs $(\mathrm{G}, \mathrm{Sc})$ where $\mathrm{G}$ is a goal and Sc a scenario. Each pair is called a requirements chunk (RC). RCs are related to one another in three different ways through composition, alternative and refinement relationships. The composition and alternative relationships lead to an AND/OR structure between $\mathrm{RCs}$ whereas the refinement relationship is used to describe RCs at different levels of abstraction (Figure 8). A brief overview of the concepts and terminology of the CREWS product model is as follows :

A Requirement Chunk (RC) is a pair $\langle\mathrm{G}, \mathrm{Sc}\rangle$ where $\mathrm{G}$ is a goal and $\mathrm{Sc}$ is a scenario. Since a goal is intentional and a scenario is operational in nature, a requirement chunk is a possible way of achieving the goal. 
A goal is defined as "something that some stakeholder hopes to achieve in the future". In our approach, a goal (similar to an intention map) is expressed as a clause with a main verb and several parameters, where each parameter plays a different role with respect to the verb. An example of a goal expressed in this structure is the following :

Provide verb (efficiently) quality (electricity) target (from EDF producer) source (to our non eligible customers) beneficiary (using the EDF network) means

A scenario is "a possible behaviour limited to a set of purposeful interactions taking place among several agents". It is composed of one or more actions, an action being an interaction from one agent to another. The combination of actions in a scenario describes a unique path. A scenario is characterised by initial and final states. An initial state attached to a scenario defines a precondition for the scenario to be triggered. A final state defines a state reached at the end of the scenario. We distinguish between normal and exceptional scenarios. The former leads to the achievement of its associated goal whereas the latter fails in goal achievement.

Classification and abstraction levels of requirement chunks: The approach recognises three levels of abstraction called contextual, functional, and physical. The contextual level identifies the services that a system should provide to an organisation and their rationale. The functional level focuses on the interactions between the system and its user to achieve the needed services. Finally, the physical level deals with the actual performance of the interactions. Each level corresponds to a type of requirement chunk. As a result, we organise the requirement collection in a three level abstraction hierarchy.

Relationships between requirement chunks: There are three types of relationships among requirement chunks namely, the composition, alternative, and refinement relationships. The first two of these lead to a horizontal AND/OR structure between RCs. These are extensions of conventional AND/OR relationships between goals. AND relationships among $\mathrm{RCs}$ link together those chunks that require each other to define a completely functioning system. RCs related through OR relationships represent alternative ways of fulfilling the same goal. The third kind of relationship relates requirement chunks at different levels of abstraction. The refinement relationship establishes a vertical link between requirement chunks.

As shown in Figure 8 the RE process is supported by automated rules embodied in a computer-based software tool called L'Ecritoire. Automated rules act in the two phases of the goal-discovery, scenario-authoring, goal-discovery cycle to respectively guide scenario authoring and help in discovering goals. 
The corresponding map and guidelines are presented in Figure 9a and Figure 9b respectively.

As can be seen, the map of Figure 9a provides a number of paths for going from Start to Stop. The sequence 'Start, linguistic strategy to Elicit a Goal, free prose to Write a Scenario, manual strategy to Conceptualize a Scenario, completeness strategy to Stop' is a path. Another path could be the one which after Conceptualize a Scenario uses the composition discovery strategy to achieve Elicit a Goal and then goes to Stop through case-based discovery to Elicit a Goal, free prose to Write a Scenario, manual strategy to Conceptualize a Scenario, completeness strategy to Stop. It is evident that each of these paths is a process model. The multiple process models that can be generated from the map are limited only by the map itself.

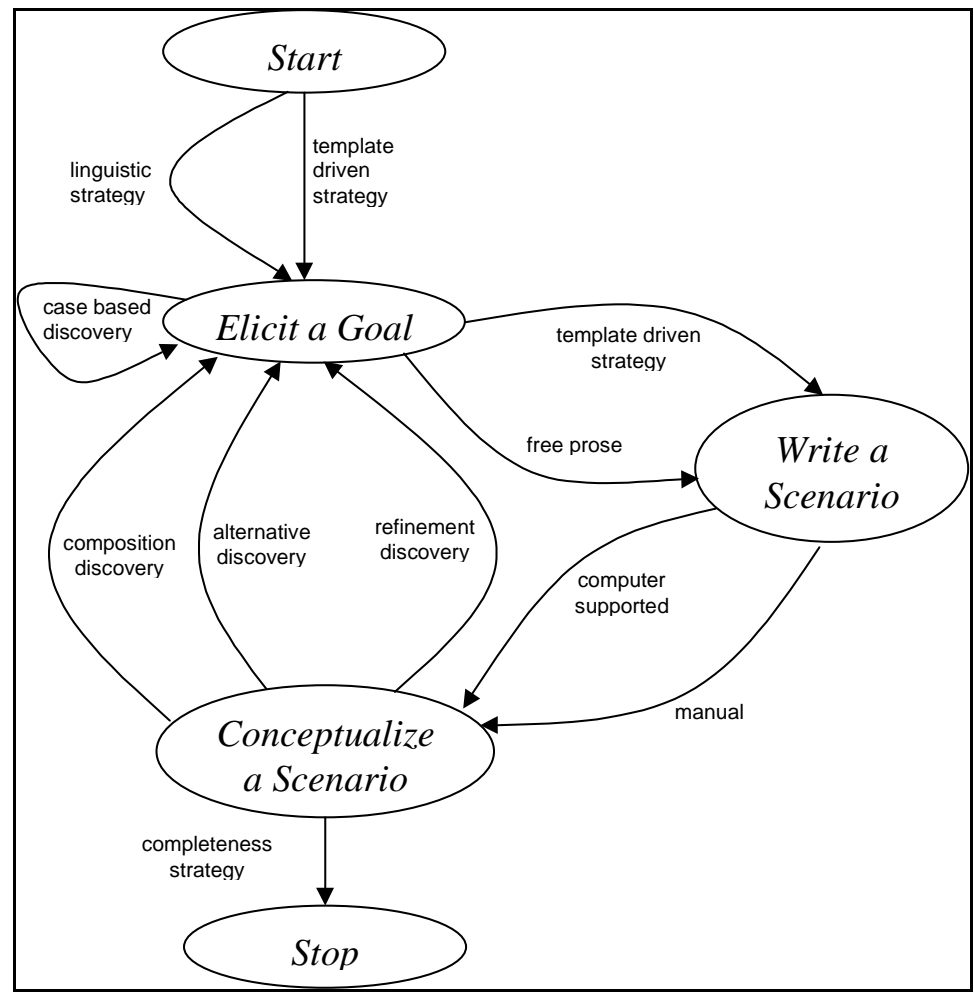

Figure 9a: Map of the CREWS-L'Ecritoire method

The generation of an actual process model is not done in any ad-hoc way but is driven by the situation of the product after an intervention has been achieved. For example, after achievement of Elicit a Goal, the situation could be that case-based discovery strategy is used to again Elicit a Goal. The resulting situation, after Elicit a Goal, could now ask for the free prose strategy to be used to Write a Scenario. The point is that the process model is shaped dynamically by the situations which arise as a result of intention achievement. This means that the time gap between process model generation and process enactment is reduced to zero. This facilitates changes in the process model as the process is performed. 
Process model generation is under the control of guidelines. For instance, SSG4 supports the selection of the linguistic strategy to Elicit a Goal in the first path presented above. ISG1 thereafter helps in the selection of Write a Scenario whereas SSG3 supports the selection of the free prose strategy for achieving it. The section (Elicit a Goal, Write a Scenario, free prose) is now selected and IAG8 supports the achievement of Write a Scenario. The use of guidelines continues till the entire process model has been generated.

\begin{tabular}{|c|c|}
\hline \multicolumn{2}{|l|}{ Intention Achievement Guidelines (IAG) } \\
\hline$<(\mathrm{G})$, Elicit a Goal with case based discovery strategy $>$ & IAG1 \\
\hline$<(\mathrm{RC}:$ state $(\mathrm{RC})=$ completed $)$, Elicit a Goal with composition strategy $>$ & IAG2 \\
\hline$<(\mathrm{RC}:$ state $(\mathrm{RC})=$ completed $)$, Elicit a Goal with alternative strategy $>$ & IAG3 \\
\hline$\langle(\mathrm{RC}:$ state $(\mathrm{RC})=$ completed $)$, Elicit a Goal with refinement strategy $>$ & IAG4 \\
\hline$<$ (Stat.), Elicit a Goal with linguistic strategy $>$ & IAG5 \\
\hline$<$ (Stat.), Elicit a Goal with template driven strategy $>$ & IAG6 \\
\hline$\langle(\mathrm{G})$, Write a Scenario with template driven strategy $>$ & IAG7 \\
\hline$\langle(\mathrm{G})$, Write a Scenario in free prose $>$ & IAG8 \\
\hline$\langle(\mathrm{Sc}$ : state $(\mathrm{Sc})=$ written $)$, Conceptualize a Scenario with computer support strategy $\rangle$ & IAG9 \\
\hline$<(\mathrm{Sc})$, Conceptualize a Scenario manually $>$ & IAG10 \\
\hline$\langle(\mathrm{RCs}:$ state $(\mathrm{RCs})=$ completed $)$, Stop with completeness strategy $>$ & IAG11 \\
\hline \multicolumn{2}{|l|}{ Strategy Selection Guideline } \\
\hline$\langle(\mathrm{RC}:$ state $(\mathrm{RC})=$ completed $)$, Progress to Elicit a Goal $>$ & SSG1 \\
\hline$\langle(\mathrm{Sc}$ : state $(\mathrm{Sc})=$ written $)$, Progress to Conceptualize a Scenario $>$ & SSG2 \\
\hline$<(\mathrm{G})$, Progress to Write a Scenario $>$ & SSG3 \\
\hline$<$ (Stat.), Progress to Elicit a Goal $>$ & SSG4 \\
\hline$\langle(\mathrm{RCs}$ : state $(\mathrm{RCs})=$ completed $)$, Progress to Stop $\rangle$ & SSG5 \\
\hline \multicolumn{2}{|l|}{ Intention Selection Guideline } \\
\hline$<(\mathrm{G})$, Progress from Elicit a Goal $>$ & ISG1 \\
\hline$\langle(\mathrm{RC}$ : state $(\mathrm{Sc})=$ completed $)$, Progress from Conceptualize a Scenario $>$ & ISG2 \\
\hline$<(\mathrm{Sc}$ : state $(\mathrm{Sc})=$ written $)$, Progress from write a Scenario $>$ & ISG3 \\
\hline$<$ (Stat.), Progress from Start $>$ & ISG4 \\
\hline
\end{tabular}

\section{Figure 9b: Guidelines of the CREWS-L'Ecritoire method}

There is an intention achievement guideline for each of the eleven sections of the map of Figure 9a. Five SSGs are associated with the five node pairs Elicit a Goal-Write a Scenario, Write a Scenario-Conceptualize a Scenario, Conceptualize a Scenario-Elicit a Goal, Start-Elicit a Goal and Conceptualize a Scenario-Stop. Additionally, there are four ISGs one for each of the map intentions, Start, Stop, Elicit a Goal and Conceptualize a Scenario. Figures 10,11 and 12 give three examples of guidelines, one for each type.

\section{$\underline{\text { IAG8 Example }}$}


As an intention achievement guideline, IAG8 provides advice to requirements engineer to achieve the goal Write a Scenario in free prose.

The guideline is characterized by its signature : $\langle$ (sit), $I\rangle$ which expresses the intention to be fulfilled (Write a Scenario in free prose) and the situation required for the intention to be fulfilled goal $(\mathrm{G})$.

The situation refers to the goal part of the product under development (i.e. the RCs hierarchy) whereas the intention is a sub-type of the Achieve signature intention of section 3. The body is a two-level hierarchy of contexts (Figure 10). The first level is a plan context suggesting two steps to write a scenario:

1. to get writing guidance if desired,

2. to write the scenario itself.

Each of these steps are component contexts of the plan. Namely $<(\mathrm{G})$, Select Writing Guidance Form>and < (G), Write a Scenario > which both offer choices.

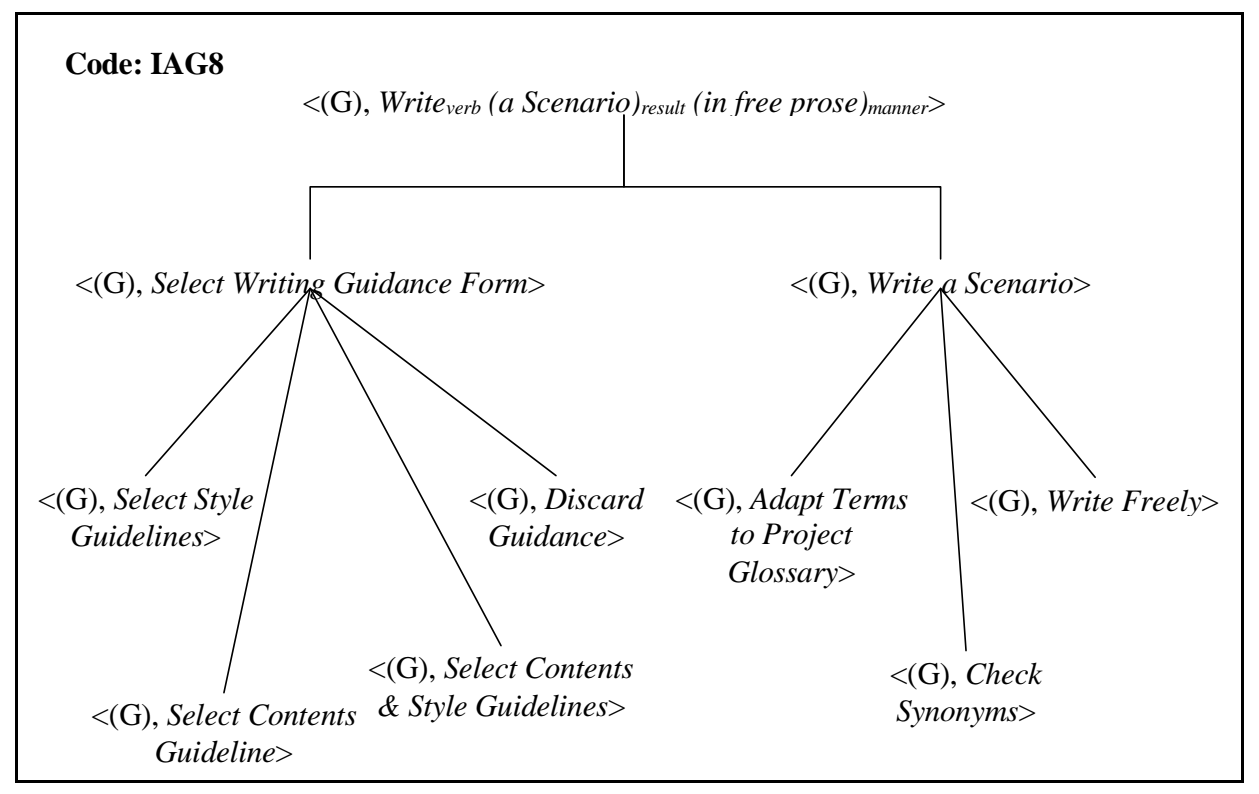

Figure 10: Example of Intention Achievement Guideline

Indeed, in the CREWS-L'Ecritoire approach, the requirements engineer has the possibility to use style guidelines, contents guidelines, both of them or to discard any proposed guidance. Style guidelines recommend a style of writing whereas contents guidelines define the semantics of the scenario contents. These choices are expressed in the choice context < (G), Select Writing Guidance Form >.

The choice context $<(\mathrm{G})$, Write a Scenario > offers three options:

(a) alignment of the terms used in the scenario with a general project glossary,

(b) detection and possible removal of synonyms,

(c) without any control.

All the leaves of the hierarchy are executable contexts. 


\section{$\underline{\text { SSG1 Example }}$}

A Strategy Selection Guideline such as SSG1 has a signature < (sit), $I>$ which expresses that the requirements engineer wants to progress in the RE process by achieving intention $I$ in a given situation (sit). The intention is a sub-type of the Progress signature intention of section 3. The SSG1 signature, < (RC: State $(\mathrm{RC})=$ completed ), Progress to Elicit a Goal> associates the intention of progressing towards the target to Elicit a Goal when the requirement chunk (RC) has been completed. Notice that in this case, the situation associates a constraint to the product part (Requirement Chunk) it refers to. The body of SSG1 is a hierarchy of contexts having the signature of SSG1 as its root. SSG1 is a choice context offering three alternatives (Figure 11). Each of these proposes the selection of an Intention Achievement Guideline to discover goals respectively following the composition strategy (Select $<(\mathrm{RC}:$ state $(\mathrm{RC})=$ completed), Elicit a Goal with composition discovery strategy $l>$ ) or the refinement strategy (Select $<$ ( $\mathrm{RC}$ : state $(\mathrm{RC})=$ completed), Elicit a Goal with refinement discovery strategy $>$ ) or the alternative strategy (Select $<(\mathrm{RC}:$ state $(\mathrm{RC})=$ completed), Elicit a Goal with alternative discovery strategy $>$ ). Arguments (a1, a2, a3) are proposed to guide the requirements engineer in the selection of the appropriate strategy and associated guideline.

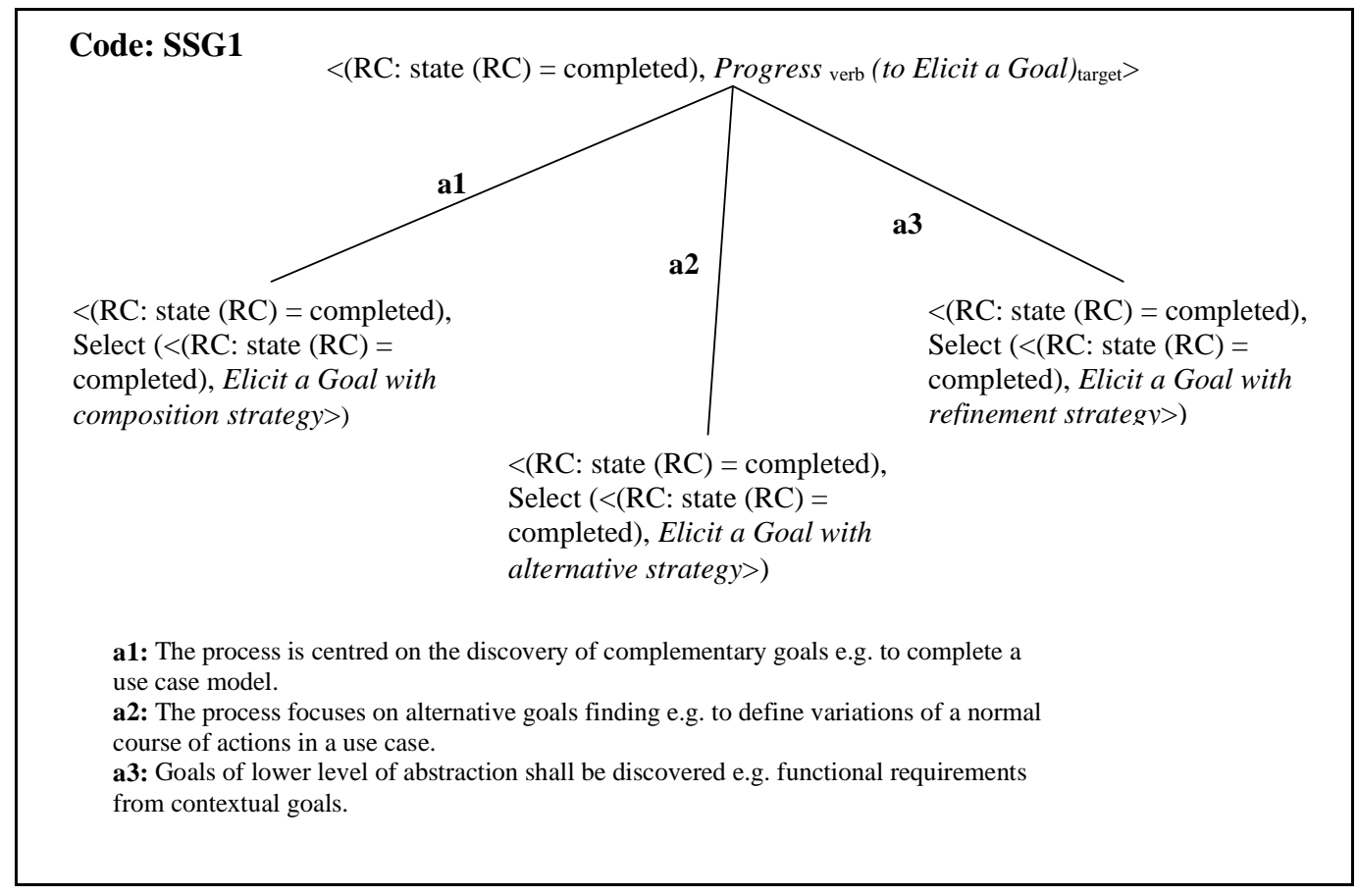

Figure 11: Example of Strategy Selection Guideline

\section{$\underline{\text { ISG1 Example }}$}

An Intention selection guideline is similar to a Strategy Selection Guideline in the sense that it guides the application engineer in progressing in the process. So, its signature 
contains an intention of the Progress type for a given situation (sit) which refers to a product part. The difference lies in the nature of the Progress intention which refers here to a "source" intention whereas it was a "target" intention in the case of a SSG. For example in ISG1, the intention is to progress from the source intention Elicit a Goal i.e. when a goal has been elicited without any specific target intention in mind.

The body of an ISG offers all the possibilities to progress from the source intention and guides in the selection of either SSGs or IAGs as described in section 3. For example, the ISG1 body (Figure 12) is a choice context which offers two alternatives: the first one suggests to proceed with the case based discovery strategy and proposes the selection of IAG1 $(<(G)$, Discover a Goal with case based discovery strategy>). The second one suggests a choice among the two strategies to Write a Scenario and proposes the selection of the SSG3 <(G), Progress to Write a Scenario $>$. Arguments a4 and a5 help in the choice of the more appropriate option for a given situation.

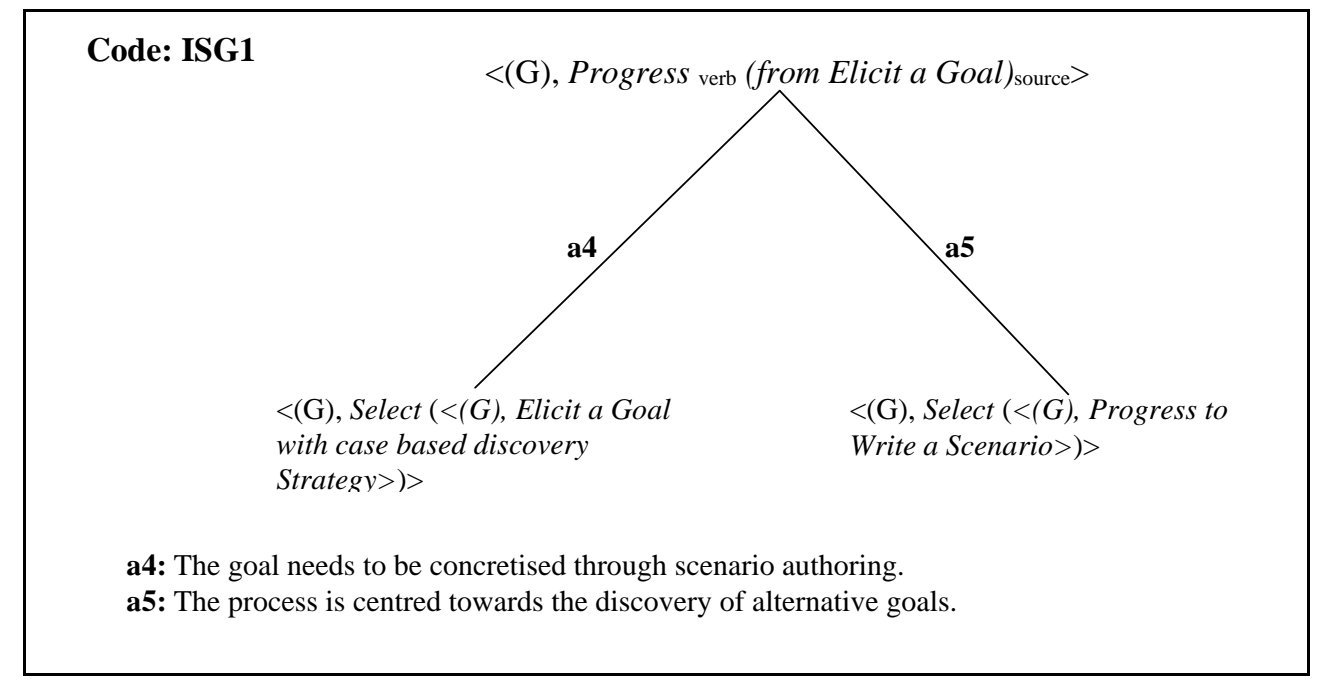

Figure 12: Example of Intention Selection Guideline

Application of the approach

Besides being applied in the CREWS-L'Ecritoire approach to requirements engineering, the multi-model view presented here has served as a basis for representing (a) the three other requirements engineering approaches developed within the CREWS project namely, the Real World Scenes approach [Hau98], the SAVRE approach for scenario exceptions discovery [Sut98] and the scenario animation approach [Dub98] and (b) for integrating approaches [Ra199] one with the other and with the OOSE approach [Jac92]. In totality this has resulted in 18 maps and almost 100 guidelines. A report on these is under preparation and is expected to be available in the electronic CREWS method base [CRI99] from September 99. 
As another important case study of the validation of the multi-model view of process modelling presented here, we would like to mention the electronic guide book to support the EKD-CM method which is a specialization of the Enterprise Knowledge Development method to managing Change Management in organisations [Nur99].

Let us now turn our attention towards the process for enacting map and guidelines i.e. the meta-process.

\section{The Meta-Process}

As in [Rol98a], we define a meta-process as a process for the construction of a process model. In our case, the meta-process is a process for the generation of a path from the map and its instantaneous enactment for the application at hand. A meta-process is an instantiation of a model, the meta-process model. The meta-process model can be represented in many different ways and we choose here the map as a means to do so. In order to avoid ambiguity we shall refer to the map of the meta-model as the meta-map and to the map of the method as the method map.

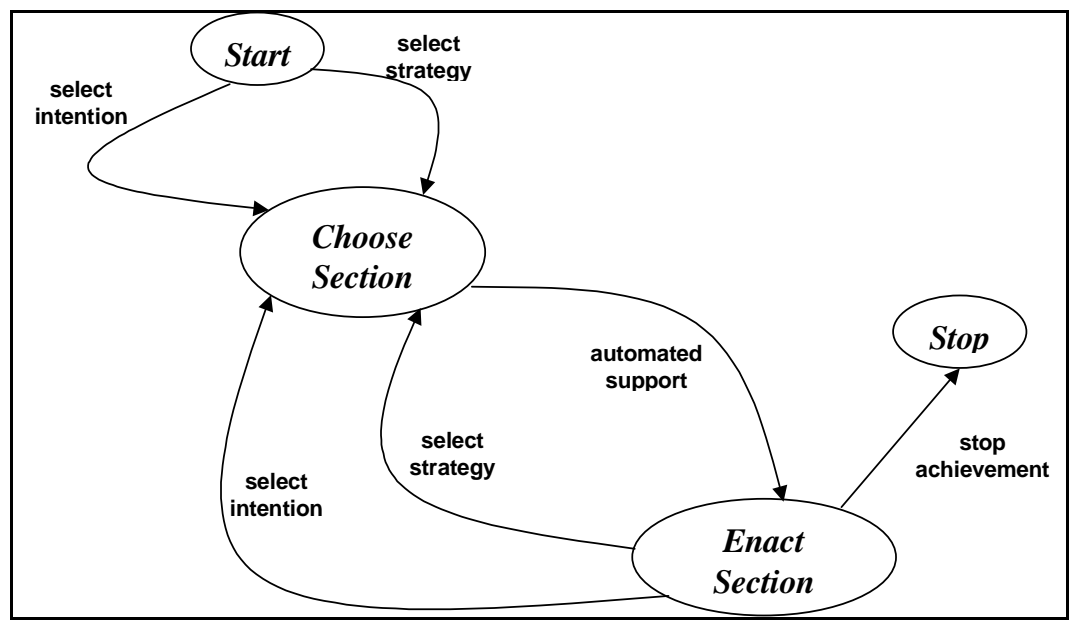

Figure 13: Meta-Process map

As shown in Figure 13, the meta-map consists of the four meta-intentions ${ }^{3}$, Start, Stop, Choose Section and Enact Section. The Start meta-intention starts the construction of a process by selecting a section in the method map which has map intention Start as source. The Choose Section meta-intention results in the selection of a method map section. The Enact Section meta-intention causes the execution of the method map section resulting from Choose Section. Finally, the Stop meta-intention stops the construction of the application process. This happens when the Enact Section metaintention leads to the enactment of the method map section having Stop as the target.

\footnotetext{
${ }^{3}$ Meta-intentions and the meta-strategies are in bold but with the fonts used for the intentions and strategies (italics and " arial" respectively).
} 
As already explained in the previous sections, there are two ways in which a section of a method map can be selected, namely by selecting an intention or by selecting a strategy. Therefore, the meta-intention Choose Section has two meta-strategies associated with it, select intention and select strategy respectively. Once a method map section has been selected by Choose Section, the IAG to support its enactment must be retrieved; this is represented in Figure 13 by associating the meta-strategy automated support with the meta-intention, Enact Section.

When these meta-strategies are used together with the meta-intentions then, six sections as shown in the figure are formed. When progressing from Start to Choose Section the application engineer can use either select intention or select strategy depending on whether the intention of the application process is unknown or the intention is known but the strategy is unknown. A similar situation occurs when progressing from Enact Section to Choose Section. There is only one strategy to proceed from Choose Section to Enact Section, namely automated support. Similarly, when Choose section progresses to Stop then the stop achievement strategy is used.

There are three key meta-IAGs for achievement of the meta-intentions. These perform the selection of the guidelines of the method map.

- ISGs for Choose section with select intention

- SSGs for Choose Section with select strategy

- IAGs for Enact Section with automated support

In the next section, we apply the meta-process model to generate a process which will produce the requirements specification of a recycling machine in a super market.

\section{A process for eliciting requirements of a recycling machine}

This section illustrates the generation of a process for the Recycling Machine (RM) case study [Jac92]. The initial situation is that of a super market wanting to provide recycling facilities to its customers. The map of the CREWS-L'Ecritoire (CL) method presented in Figure 9a is used by the meta-process to elicit the requirements of this machine. This method map will be referred to in the following as the CL map.

The meta-process is used to drive the selection of the appropriate section in the CL map and to enact the CL guidelines in order to elicit the requirements for the RM. Figure 14 highlights the 8 sections of the CL map selected and enacted as examples of the process 
steps for the RM. These sections are sequentially numbered according to the order tin which they are selected and enacted.

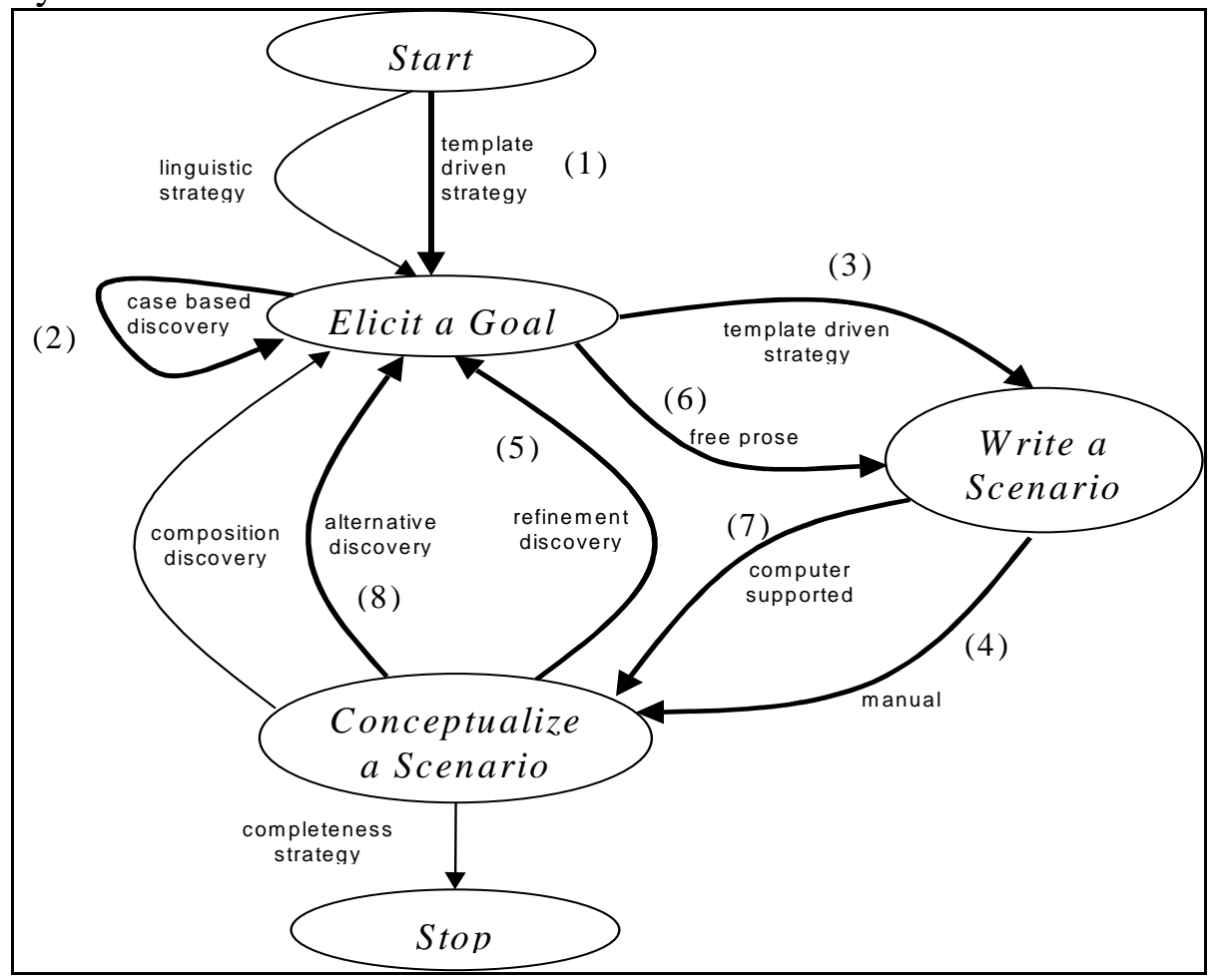

Figure 14: Use of CL map for RM Example

Figure 15 shows the corresponding sequence of sections in the meta-map. Clearly each step of the RM process results from two iterations in the meta-map : one to guide the selection of the appropriate section in the CL map for the situation at hand and the other one to guide the enactment of the IAG associated to the CL selected section (denoted $\mathrm{n} .1$ and $\mathrm{n} .2$ respectively for any step $\mathrm{n}$ in Figure 15). The trace of the eight steps in both the meta-process and of the process is shown in Table1. In the following we explain the interaction between the meta-process, the CL map and the requirements engineer for the first process step. The other steps shall be interpreted from Table1 in the same way. 


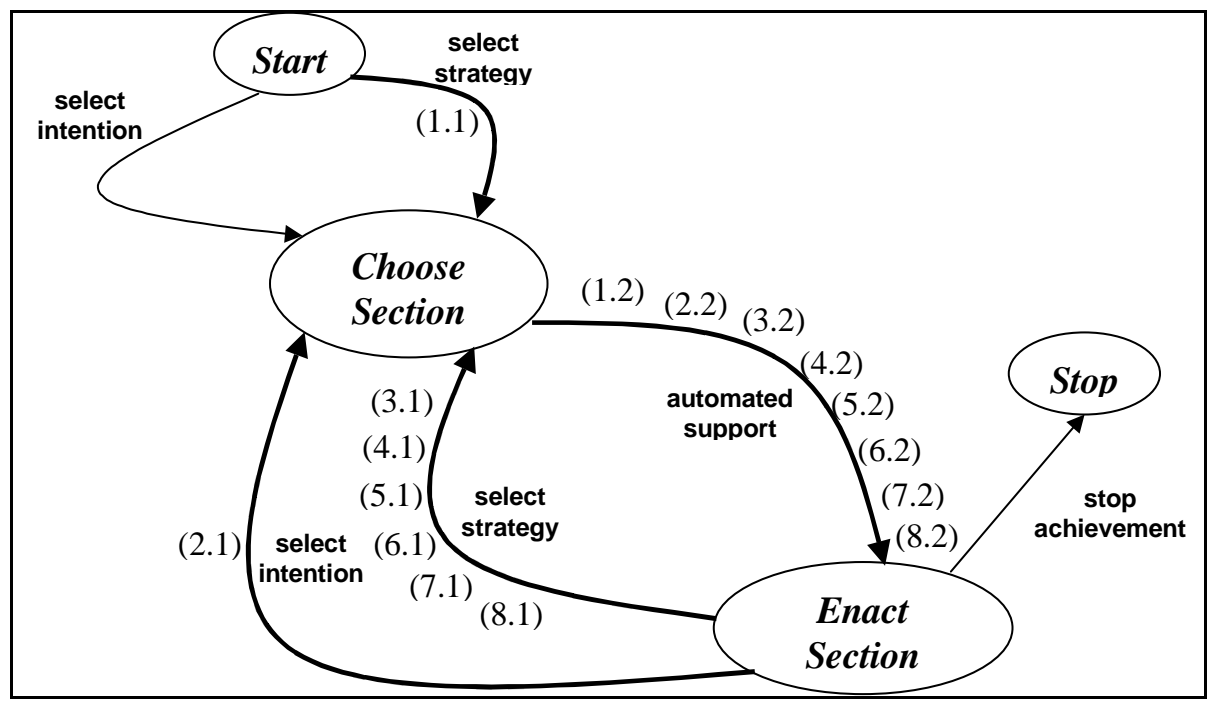

Figure 15: Use of meta-process for RM Example

The meta-process begins from the meta-intention Start. In the CL map there is exactly one intention, namely Elicit a Goal with Start as a source. Therefore, the meta-strategy is clearly select strategy to Choose Section (see Figure 15). The achievement of the Choose Section following select strategy leads to the presentation of the SSG4 guideline (column 1 in the first raw of Table1) to the requirements engineer. The argument used by the requirements engineer to select from the choices offered by SSG4 is shown in the second column of the first row of Table 1. The result of this is the selected section shown in the third column of this row. This explains how the meta-map helps the requirements engineer selecting a section in the CL map. It is summarised in the first raw of step1 in Table1.

Now, in the meta-process the next meta-intention is Enact Section (see Figure 15) which is to be achieved by using the automated support meta-strategy. In the CL map this results in the selection of the IAG6 guideline that is displayed to the requirements engineer. This is shown in column 1 of the second row in Table1. The enactment of this guideline is discussed in the second column of the second row of the table. The impact of this enactment on the product is shown in the last column of this row.

Thus the second raw in Table1 for a given step sums up the effect of enacting the IAG guideline corresponding to the section selected in the first raw of the table for this step.

Now, in the meta-process, the next meta-intention is Choose Section with one of the two meta-strategies select strategy and select intention. This starts step 2 in the RM process. Since in the CL map there are two intentions which can be achieved, the metastrategy selected is select intention (see Figure 15). As traced in the first column of the first raw for step 2 in Table1, this selection results in an achievement of the Choose 
Section leading to the presentation of the ISG1 guideline to the requirements engineer. The argument used by the requirement engineer is shown in the second column of this row of the table and the resulting selected section is shown in the last column.

In this way, the interaction of the meta-process, the CL map and the application engineer continues. Eight iterations in the meta-process are shown in Table 1. These generate a partial specification of the RM.

\begin{tabular}{|c|c|c|c|}
\hline \multirow{2}{*}{$\begin{array}{c}\text { Step } \\
\text { Number } \\
\end{array}$} & Meta-Process & \multicolumn{2}{|c|}{ Process } \\
\hline & $\begin{array}{c}\text { Column } 1 \\
\text { Displayed guidelines }\end{array}$ & $\begin{array}{c}\text { Column } 2 \\
\text { IS \& SS Guidelines Arguments }\end{array}$ & $\begin{array}{c}\text { Column } 3 \\
\text { Selected section }\end{array}$ \\
\hline \multirow[t]{4}{*}{1} & $\begin{array}{l}\text { Iteration } 1.1 \\
\text { Choose section with select } \\
\text { strategy }\end{array}$ & $\begin{array}{l}\text { SSG4 suggests two strategies. } \\
\text { The template driven strategy is chosen } \\
\text { because it is the most appropriate way } \\
\text { to get familiar with the goal } \\
\text { formalization proposed by the CREWS } \\
\text { L'Ecritoire method. }\end{array}$ & $\begin{array}{l}\text { Start, } \\
\text { Elicit a Goal, } \\
\text { template driven strategy) }\end{array}$ \\
\hline & \multirow[b]{2}{*}{$\begin{array}{l}\text { Iteration } 1.2 \\
\text { Enact section with } \\
\text { automated support }\end{array}$} & IA Guidelines Arguments & Product \\
\hline & & $\begin{array}{l}\text { IAG6 displays a goal statement } \\
\text { template and explains the meaning of } \\
\text { each parameter. The requirement } \\
\text { Engineer (RE) chooses a loose } \\
\text { statement having only a verb and a } \\
\text { target. }\end{array}$ & $\begin{array}{l}\text { G1: } \\
\text { Provide }{ }_{\text {verb }}(\text { Recycling } \\
\left.\text { Facilities }^{*}\right)_{\text {target }}\end{array}$ \\
\hline & Displayed guidelines & IS \& SS Guidelines Arguments & Selected section \\
\hline 2 & $\begin{array}{l}\text { Iteration } 2.1 \\
\text { Choose section with select } \\
\text { intention }\end{array}$ & $\begin{array}{l}\text { ISG1 provides RE with arguments to } \\
\text { advise him on choosing one of the two } \\
\text { possible intentions from Elicit a goal } \\
\text { namely to Elicit a goal or to Write a } \\
\text { Scenario. The former is selected so as } \\
\text { to generate alternative design } \\
\text { solutions. }\end{array}$ & $\begin{array}{l}\text { (Elicit a Goal, } \\
\text { Elicit a Goal, } \\
\text { case based strategy) }\end{array}$ \\
\hline
\end{tabular}




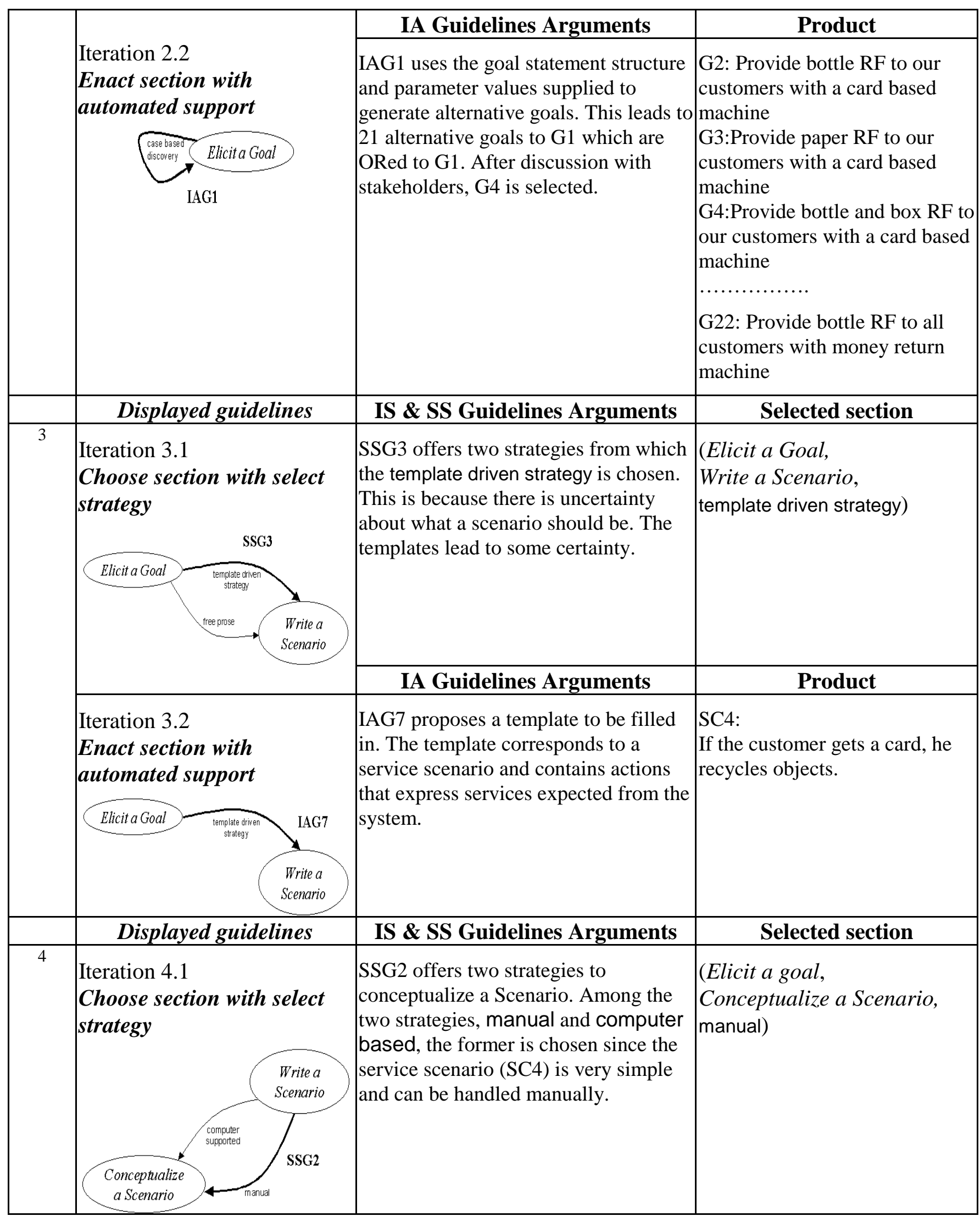




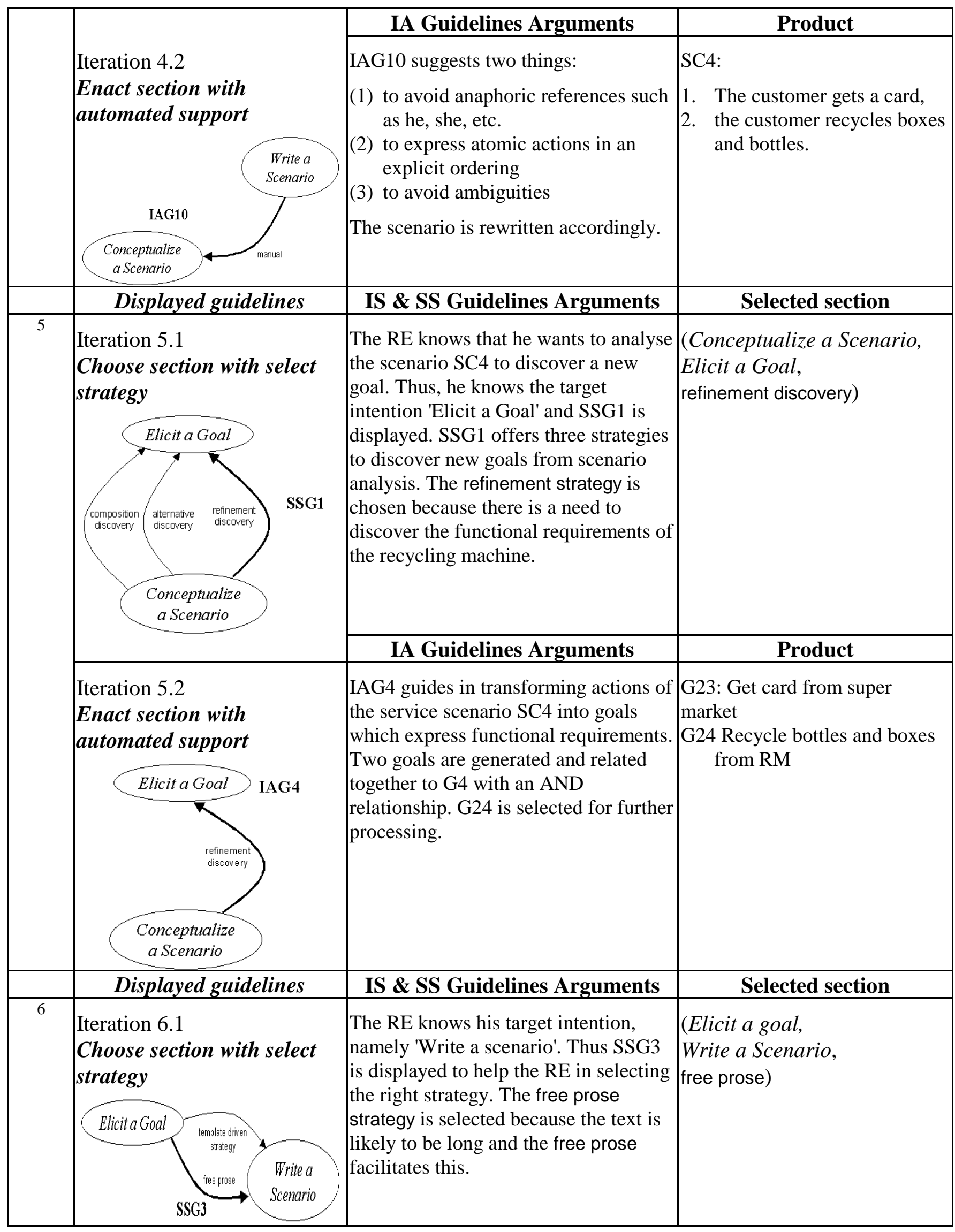




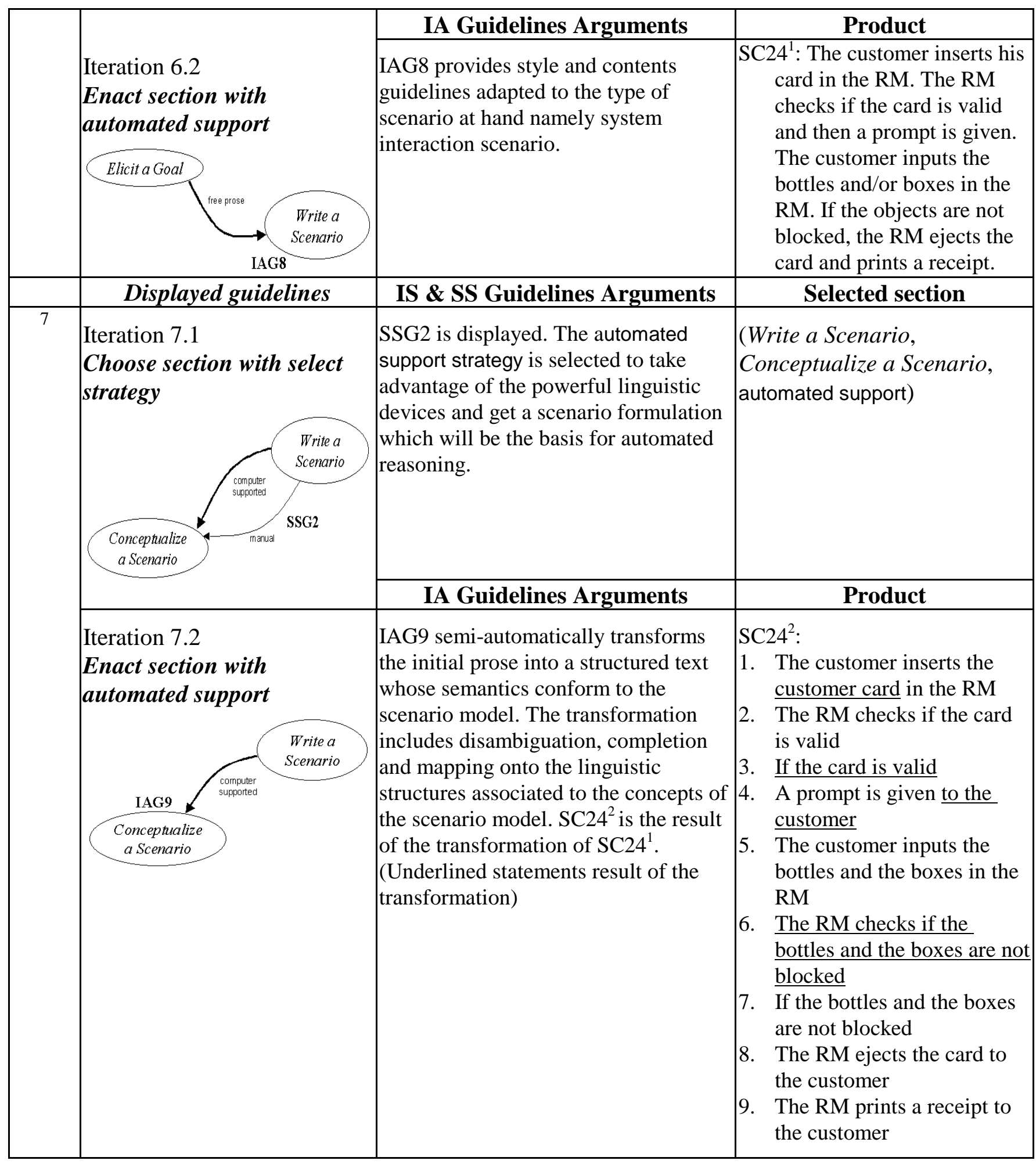




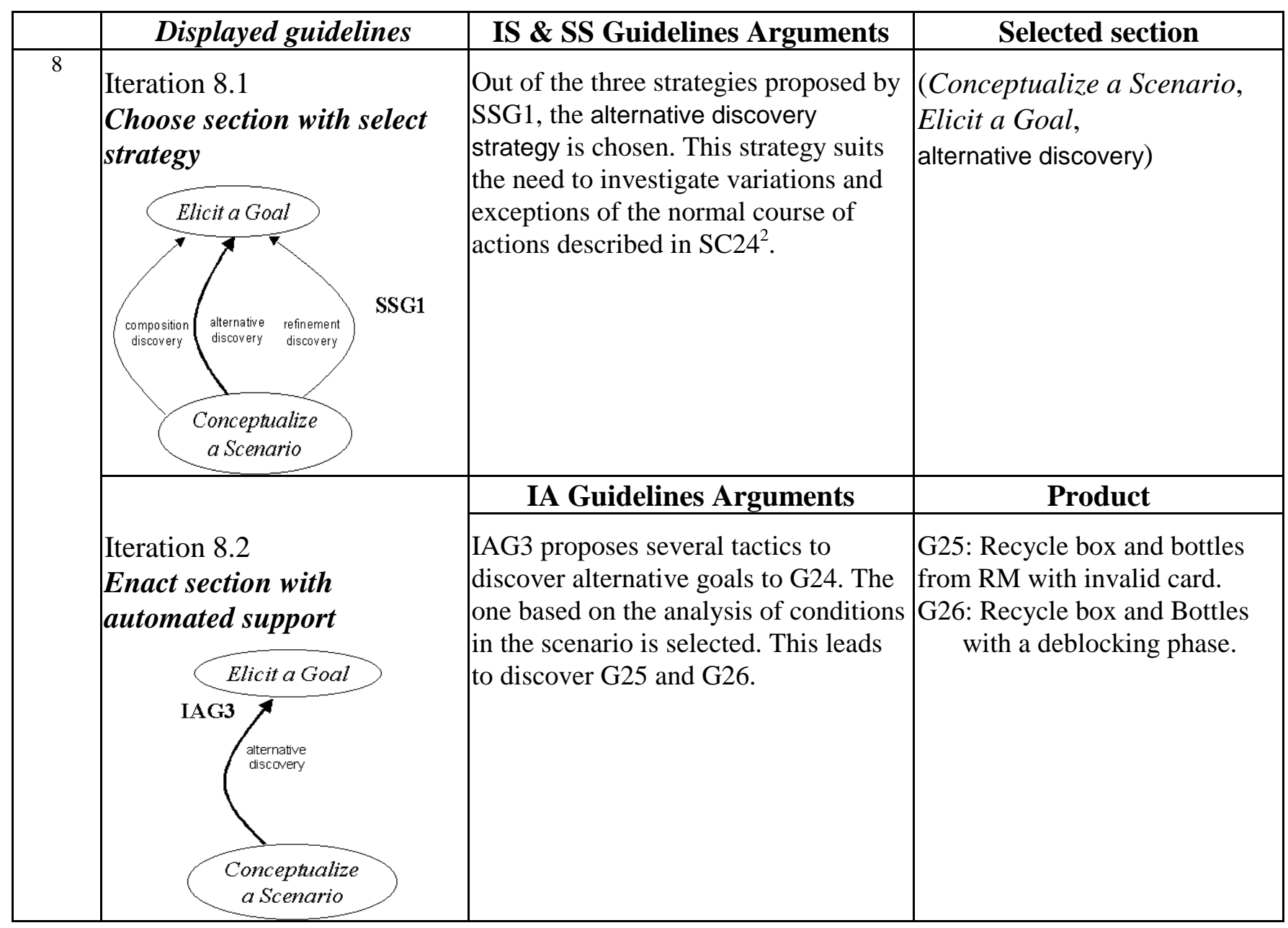

Table 1 : Trace of the process to elicit requirements for the Recycling Machine case study

The arguments contained in column 2 of the table show the use of non-determinism in intention and strategy selection embodied in the map. It also shows that for a given type of situation different strategies are chosen for different situations (instances) of this type. This effect is seen in iterations 3 and 6, 4 and 7 as well as in 5 and 8 .

\section{Conclusion}

Early process models presented a take it or leave it choice to application engineers, either you adopted a certain model or you discarded it and chose another one. However, the recognition of the role of process situations in shaping the process model has resulted in adapting process models to situational needs. The basic approach to process modelling has however remained the same: process models are statically defined even though they are expected to handle dynamically changing situations. In other words, knowledge of all situations likely to occur is assumed to be statically available. This is 
clearly an untenable assumption.

Our approach is to respond to a dynamically changing situation by constructing process models dynamically. As a result, the process model handles a situation as it emerges and it is completely sensitive to the situation at all times.

Prevalent approaches to process modelling emphasize task organization and are therefore principally concerned with the tactics to be adopted in carrying out the task. In the multi-model view presented here, we have called for a shift to the relatively more upstream activities performed to develop real processes, those of deciding what is to be done (intentions) and the manner (strategies) in which this is to be done. Thus, our focus is on strategic issues concerning process modelling. In fact, we separate the strategic from the tactical by representing the former in the method map and embodying the latter in the guidelines. By associating the guidelines with the map, a smooth integration of the strategic and the tactical aspects is achieved.

The capability to dynamically construct process models provided in the multi-model view is directly related to the identification of intentions and strategies needed. The dynamicity is promoted by the fine-grained modularity of sections and their high interconnectivity. This encourages flexible manœuvrability in constructing multiple paths from the map. 


\section{VIIIReferences}

[Aae92] Aaen et Al, A tale of two countries:CASE experience and expectations, The Impact of Computer Supported Technology on Information Systems Development, North Holland Pub, pp 61-93, 1992.

[Arm93] P. Armenise, S. Bandinelli, C. Ghezzi, A. Morzenti, A survey and assessment of software process representation formalisms Int. Journal of Software Engineering and Knowledge Engineering, Vol. 3, No. 3, 1993.

[Ban93] S. Bandinelli, A. Fugetta, S. Grigoli, Process Modelling in the large with SLANG. Proceedings of the 2nd International Conference on "Software Process ", Berlin, GERMANY, 1993, pp 75-93.

[Be194] N. Belkhatir, W. L. Melo, Supporting Software Development Processes in Adele2. The Computer Journal, vol 37, N², 1994, pp 621-628.

[Ben98] C. Ben Achour, Guiding scenario authoring. Proceedings of the 8th European Japanese Conference on "Information Modelling and Knowledge Bases ", pp 181-200, Ellivuori, FINLAND, May 26-29, 1998.

[Bub94] J. Bubenko, C. Rolland, P. Loucopoulos, V De Antonellis, Facilitating 'Fuzzy to Formal' requirements modelling. IEEE 1st Conference on "Requirements Enginering " (ICRE'94), pp. 154-158, 1994.

[CRI99] www.univ-paris1.fr/CRINFO/METHODBASE/

[Cug95] G. Cugola, E. Di Nitto, C. Ghezzi, and M. Mantione, How to deal with deviations during process model enactment. Proceedings of 17th International Conference on "Software Engineering” (ICSE17), Seattle, Washington, USA, April 1995.

[Cug96] G. Cugola, E. Di Nitto, A. Fuggetta, and C. Ghezzi, A Framework for Formalizing Inconsistencies and Deviations in Human-Centered Systems. ACM Transactions on "Software Engineering and Methodology" (TOSEM), vol. 5, num. 3, July 1996.

[Cug98] G. Cugola, Inconsistencies and Deviations in Process Support Systems. Ph.D. Thesis Politecnico di Milano, February 1998.

[Dow93] M. Dowson, Software Process Themes and Issues, IEEE 2nd Int. Conf. on the Software Process, pp 28-40, 1993.

[Dow94] M. Dowson, C. Fernstrom, Towards requirements for Enactement Mechanisms. Proceedings of the 2th European Workshop on "Software Process Technology", 1994

[Dub98] E. Dubois, P. Heymans, Scenario-Based Techniques for Supporting the Elaboration and the Validation of Formal Requirements, Submitted to Requirement Engineering Journal, 1998.

[Fin94] A. Finkelstein, J. Kramer, B. Nuseibeh (eds), Software Process Modelling and Technology. John Wiley (pub), 1994.

[Gha97] S. A. Ghannouchi, H. H. Ben Ghesala, Utilisation du méta-modèle NATURE pour modéliser le processus de retro-conception de données. Proceedings of 
the 1st International Workshop on the "Many Facets of Process Engineering", Gammarth, TUNISIA, 22-23 September 1997.

[Gro97] G. Grosz, C. Rolland, S. Schwer, C. Souveyet, V. Plihon, S. Si-Said, C. Ben Achour, C. Gnaho, Modelling and Engineering the Requirements Engineering Process : an overview of the NATURE approach. Requirements Engineering Journal 2, pp. 115-131, 1997.

[Har94] Harmsen A.F., Brinkkemper J.N., Oei J.L.H.; Situational Method Engineering for information Systems Project Approaches, Int. IFIP WG8. 1 Conf. in CRIS series : Methods and associated Tools for the Information Systems Life Cycle (A-55), North Holland (Pub. ), 1994.

[Hau98] Peter Haumer, Klaus Pohl, Klaus Weidenhaupt, Requirements Elicitation and Validation with Real World Scenes, to appear in IEEE Transactions on Software Engineering, Vol. 24, No. 12, Special Issue on Scenario Management, December 1998.

[Hid94] Hidding G.J., Methodology information : who uses it and why not?, Proc. WITS-94, Vancouver, Canada, 1994.

[Jac92] I. Jacobson, M. Christerson, P. Jonsson, G. Oevergaard, Object Oriented Software Engineering: a Use Case Driven Approach, Addison-Wesley, 1992

[Jar94] M. Jarke, K. Pohl, C. Rolland, J. R. Schmitt, Experienced-Based Method Evaluation and Improvement : A Process Modeling Approach, Int. IFIP WG8. 1 Conf. in CRIS series : Method and associated Tools for the Information Systems Life Cycle, North Holland (Pub. ), 1994.

[LPR95] Le Petit Robert, French Dictionary, 1995.

[Nur99] S. Nurcan, C. Rolland, Using EKD-CMM electronic guide book for managing change in organisations, Submitted to the European-Japanese Conference on "Information Modelling and Knowledge Bases ", Japan, 1999.

[Pli94] V. Plihon. The OMT, OOA, SA/SD, E/R, $O *$, OOD methodologies, NATURE Deliverable DP2, 1994.

[Po196] K. Pohl. PRO-ART: An Environment for Enabling Requirements Traceability. Proceedings of the 2nd International Conference on "Requirements Engineering ", Colorado Springs, Colorado, USA, 1996.

[Pra97] N. Prat, Goal Formalisation and classification for Requirements Engineering. Proceedings of the Third International Workshop on "Requirements Engineering: Foundations of Software Quality" (REFSQ'97), Barcelona, SPAIN, pp. 145-156, June 1997.

[Ra199] J. Ralyté, C. Rolland, V. Plihon, Method Enhancement by Scenario Based Techniques, To appear in the proceedings of the 11th Conference on Advanced Information Systems Engineering, Heidelberg, Germany, June 14-18, 1999.

[Ro193] C. Rolland, Modelling the Requirements Engineering Process. 3rd EuropeanJapanese Seminar on "Information Modelling and Knowledge Bases", Budapest, HUNGARY, June 1993.

[Ro194a] C. Rolland, G. Grosz, A General Framework for Describing the Requirements Engineering Process. IEEE Conference on "Systems, Man and Cybernetics" 
(CSMC94), San Antonio, Texas, USA, 1994.

[Rol94b] C. Rolland, N. Prakash, Guiding the Requirements Engineering Process. Proceedings of the IEEE Asia-Pacific Software Engineering Conference (APSEC), Tokyo, JAPAN, 1994.

[Ro195] C. Rolland, C. Souveyet, M. Moreno, An Approach for Defining Ways-OfWorking. Information Systems Journal, , Vol 20, No 4, pp337-359, 1995.

[Ro197] C. Rolland, C. Ben Achour, Guiding the construction of textual use case Specifications. Data \& Knowledge Engineering Journal Vol. $25 \mathrm{~N}^{\circ} 1$, pp. 125160, North Holland, Elsevier Science Publishers, 1997.

[Ro198a] C. Rolland, A Comprehensive View of Process Engineering. Proceedings of the 10th International Conference CAiSE'98, B. Lecture Notes in Computer Science 1413, Springer Verlag Pernici, C. Thanos (Eds), Pisa, ITALY, June 1998.

[Ro198b] C. Rolland, C. Souveyet, C. Ben Achour. Guiding Goal Modelling using Scenarios, IEEE Transactions on Software Engineering, Special Issue on Scenario Management, Vol. 24, No. 12, 1055- 1071, Dec. 1998.

[Rus95] Russo, The use and adaptation of system development methodologies, Proceedings 1995 International Resources Management. Association Conference, Atlanta, USA, 1995

[SiS96] S. Si-Said, C. Rolland, G. Grosz, MENTOR :A Computer Aided Requirements Engineering Environment. Proceedings of CAiSE' 96, Crete, GREECE, May 1996.

[SiS97] S. Si Said, Guidance for requirements engineering processes. Proceedings of the 8th International Conference and Workshop on "Database and Experts System Application ” DEXA'97, Toulouse, FRANCE, 1-5 September 1997.

[Sut97] A. G. Sutcliffe, M. Jarke, C. Rolland, J. Bubenko, P. Constantopoulos. Defining visions in context models, process and tools for Requirements Engineering. Information Systems Journal, Volume 21, no:6, pp. 515-547, 1997.

[Sut98] A.G. Sutcliffe, N.A.M. Maiden, S. Minocha, D. Manuel, Supporting Scenariobased Requirements Engineering, Transaction of Software Engineering: Special Issue on Scenario Management, 1998.

[Wel92] Welke R.J, and Kumar K., Method engineering : a proposal for situationspecific methodology construction, in Systems Analysis and Design. A Research Agenda, Cotterman and Senn(eds), Wiley, pp257-268, 1992.

[Wij90] Wijers G. M., van Dort H.E., Experiences with the use of CASE tools in the Netherlands, Advanced Information Systems Engineering, pp 5-20, 1990.

[Your92] Yourdon E., The decline and fall of the american programmer, Prentice Hall, Englewood Cliffs, NJ, 1992. 